\title{
Characterization of Fabry-Perot interferometers and multi-etalon transmission profiles
}

\section{The IBIS instrumental profile}

\begin{abstract}
K. P. Reardon and F. Cavallini
INAF - Osservatorio Astrofisico di Arcetri, Largo E. Fermi 5, 50125 Firenze, Italy

e-mail: kreardon@arcetri.astro.it

Received 13 August 2007 / Accepted 16 January 2008

ABSTRACT

Aims. Properly characterizing Fabry-Perot interferometers (FPI) is essential for determining their effective properties and evaluating the performance of the astronomical instruments in which they are employed. Furthermore, in two-dimensional spectrographs where multiple FPI are used in series, the actual distribution of plate separation errors will be crucial for determining the resulting transmission profiles. We describe techniques that address these issues utilizing the FPI of IBIS, a solar bidimensional spectrometer installed at the Dunn Solar Telescope.

Methods. A frequency-stabilized He-Ne laser was used in three different optical layouts to measure the spatially-resolved transmission of the FPI. Analyzing the shape and wavelength shift of the observed profiles allows the characteristics of the cavity errors and the interferometer coating to be determined.

Results. We have measured the spatial distribution of the large-scale plate defects, which shows a steep radial trend, as well as the magnitude of the small-scale microroughness. We also extracted the effective reflectivity and absorption of the coating at the laser line wavelength for both interferometers.

Conclusions. These techniques, which are generally applicable to any Fabry-Perot interferometer, provide the necessary information for calculating the overall instrumental profile for any illuminated area of the interferometer plates. Accurate knowledge of the spectral transmission profile is important, in particular when using inversion techniques or in comparing observations with simulated data.
\end{abstract}

Key words. instrumentation: interferometers - instrumentation: spectrographs - Sun: general - methods: laboratory

\section{Introduction}

The Fabry-Perot interferometer (FPI) has many desirable properties that make it very suited for use in a range of scientific instrumentation. The FPI can be used to obtain monochromatic images over a full two-dimensional field of view with spectral resolutions comparable to those of grating spectrographs. Moreover, due to its large area and to dielectric multilayer coatings with very low absorption coefficients, this device has high throughput and transmittance, which make it suitable for high temporal resolution and detection of faint objects.

For these reasons the FPI is often in use in night-time instruments, such as 3DII (Ishigaki et al. 2004) on Subaru, OSIRIS (Cepa et al. 2003) on GCT and PFIS (Burgh et al. 2003) on SALT. This latter, as well as the proposed MSASI instrument (Yoshikawa et al. 2007) for BepiColombo, use two FPI in series to achieve a higher spectral resolution with a larger free spectral range. In solar astronomy, instruments have been constructed either combining a single FPI with a birefringent filter (Bendlin \& Volkmer 1995; Cavallini 1998; Denker et al. 2003) or based on two or more FPI in series to form a multi-etalon system for solar bidimensional spectroscopy and/or spectropolarimetry (Ramsay et al. 1970; Kentischer et al. 1998; Puschmann et al. 2006; Cavallini 2006).

The modern piezo-scanned and capacity servo-controlled FPI are affordable, robust devices, able to be rapidly tuned in wavelength, with a linear response and without hysteresis effects, with a stable and accurate parallelism of the plates.
However, one possible drawback of this device is its high sensitivity to the small defects in the reflecting surfaces, due to the high number of multiple reflections within the optical cavity. The transparency profile and, in classic mount, the image quality, are affected by the flatness errors of the interferometer plates. The measurement of the cavity errors and their spatial distribution will allow their proper consideration in the design phase, reducing their overall impact on the instrumental performance.

Moreover, knowledge of the cavity errors present in the FPI allows the overall spectral transmission profile, or instrumental profile, to be deduced. This is important for the retrieval of accurate physical information from the observed spectral profiles. In solar physics this is often achieved through the comparison of the acquired data to numerical simulations (Cauzzi et al. 2006), or through the application of inversion techniques for spectropolarimetry (Skumanich \& Lites 1987; Socas-Navarro 2001, 2003). In both cases an accurate knowledge of the instrumental profile is required.

It is relatively straightforward to measure the transmission profile for one FPI at a single wavelength with a laser (Anderson 1995; Gullixson 1998; Cao et al. 2004; Denker \& Tritschler 2005), but current instruments generally employ multiple FPI and operate over a broad range of wavelengths. To calculate the instrumental profile in this case, a comprehensive understanding of the physical characteristics of the interferometers is necessary. In addition, in the presence of cavity errors with large spatial scales, a more complicated procedure to calculate the 
instrumental profile must be followed than the simple multiplication of integrated transmission profiles for each FPI.

In this paper we describe the techniques for the characterization of a Fabry-Perot interferometer, in particular the methods to measure both the spatial distribution of the large-scale plate separation errors and magnitude of the randomly distributed smallscale errors down to the level of the unresolved microroughness. From these measurements we are also able to provide estimates of the coating reflectivity and absorption.

We apply these general purpose techniques to the two FPI used in the Interferometric BIdimensional Spectrometer (IBIS), a solar imaging spectrometer installed at the Dunn Solar Telescope of the National Solar Observatory, which uses the FPI in a classic mount and operates over the range 5800$8600 \AA$ (for further details, see Cavallini 2006, hereinafter referred to as Paper I). The results of these measurements are used to calculate the overall instrumental profile of IBIS and to show its effect on the simulated observations of synthetic spectral lines.

The knowledge of the magnitude and distribution of the plate defects is also important in the determination of how they might reduce the optical quality due to deformations of the wavefront shape and pupil apodization effects (Ramsay 1969; Beckers 1998; von der Lühe \& Kentischer 2000; Scharmer 2006). In a following paper, these same measurements will be used to evaluate the effects of the cavity errors on the image quality and to compare theoretical and measured MTF for IBIS.

\section{Data description}

Our approach in characterizing the IBIS interferometers was to make spatially resolved measurements of the transmission profiles over an area illuminated by a stabilized He-Ne laser (Table 1). For this single mode laser, the width of the emission profile is much less than $1 \mathrm{~m} \AA$, and hence much narrower than the transmission profiles we are attempting to measure (20$40 \mathrm{~m} \AA$ ). The laser operates at a fixed wavelength and the spectral scanning has thus been performed by changing the wavelength position of the interferometer transmission profile through incremental increases in the plate spacing. In this way, as we increased the interferometer spacing and moved the transmission profile past the laser emission peak from the blue to the red, the interferometer profile was sequentially sampled from longer to shorter wavelengths (Wilksch 1985).

The two IBIS FPI (see Table 2) are piezo-electrically tuned and the spacing and the parallelism of their plates are maintained by means of a CS100 electronic controller. Moreover, to eliminate the effect of changes in the ambient pressure, temperature and humidity on the capacitance sensors and on the length of the optical cavity, each interferometer is enclosed in a sealed cell and is thermally controlled within $\pm 0.005^{\circ} \mathrm{C}$, maintaining the wavelength drift of the transmission profile to less than $1 \mathrm{~m} \mathrm{~s}^{-1}$ over $1 \mathrm{~h}$ (Paper I). Both FPI have wedged plates to eliminate spurious reflections from the rear surfaces.

The CS100 has a digital resolution of 12 bits, allowing the changing of the applied voltage by discrete steps of $1 \mathrm{~V}$, corresponding to nominal step size of $4.8 \AA$. We determined the actual step size for each FPI by measuring the number of voltage steps between adjacent peaks of the transmission profile using the laser. Since the overall increase in plate separation between peaks is $\lambda / 2$, we were able to determine that the dispersion for FPI \#1 (see Table 2) was $4.80 \AA$ per step, and $4.59 \AA$ for FPI $\# 2$. For an interferometer with a nominal plate separation $t$, the
Table 1. Laser characteristics.

\begin{tabular}{ll}
\hline \hline Model & Spectra-Physics 117 \\
Frequency (nominal) & $473.61254 \mathrm{THz}(6328.1 \AA)$ \\
Beam diameter $\left(1 / \mathrm{e}^{2}\right)$ & $0.5 \mathrm{~mm}$ \\
Beam divergence & $1.6 \mathrm{mrad}$ (full angle) \\
Frequency stability & $\pm 2 \mathrm{MHz}\left( \pm 1.27 \mathrm{~m} \mathrm{~s}^{-1}\right)$ \\
& $1-8 \mathrm{~h}($ guaranteed) \\
Intensity stability & $1 \%$ \\
\hline
\end{tabular}

Table 2. Fabry-Perot interferometer characteristics.

\begin{tabular}{|c|c|}
\hline Manufacturer & IC Optical Systems \\
\hline Type & ET50 FS \\
\hline Clear aperture $(\mathrm{mm})$ & 50 \\
\hline Plate spacing (mm) & 2.300 (FPI \#1), 0.637 (FPI \#2) \\
\hline Wedge angle & $20^{\prime}$ \\
\hline Coating & Multilayer broadband \\
\hline Dispersion $(\AA /$ step $)$ & 4.80 (FPI \#1), 4.59 (FPI \#2) \\
\hline Wavelength range $(\AA)$ & $5800-8600$ \\
\hline Nominal reflectance (@6328 A) & 0.942 \\
\hline Nominal absorption coefficient & $\leq 0.002$ \\
\hline (@6328 A) & $\lambda / 150$ (after coating) \\
\hline
\end{tabular}

relationship between the change in spacing $(\Delta t)$ and the wavelength shift of the transmission profile $(\Delta \lambda)$ is given by:

$\Delta \lambda=\lambda \frac{\Delta t}{t}$

At the laser wavelength (6328.1 $\AA$ ), FPI \#1 has the smaller wavelength step of $1.32 \mathrm{~m} \AA$, while FPI \#2, with the smaller value of $t$, has a nominal step of $4.56 \mathrm{~m} \AA$.

We used two different layouts, collimated (Fig. 1) and telecentric (Fig. 9), in order to characterize the interferometers. A third configuration, the classic mount (Fig. 13), was used to verify the results of the measurement. The list of the scans obtained is shown in Table 3.

The data were recorded with a Princeton Instruments PentaMAX camera with a dynamic range of 12 bits and $18 \mathrm{e}^{-}$ (1.8 ADU) rms read noise. The CCD detector was a Kodak KAF-1400 with $1317 \times 1035$ pixel with physical dimensions of $6.8 \times 6.8 \mu \mathrm{m}^{2}$ each. Each recorded image was the sum of 5 images taken in succession and coadded by the camera acquisition software. Typical signal levels ranged from a peak of 15000 down to about 30 ADU $\left(150000-300 \mathrm{e}^{-}\right)$.

All the data were corrected with dark current and bias measurements as well as for the non-linear response of the CCD. Weak high-frequency spatial fringes, present in some images, were removed with a suitable Fourier filter. The images were flatfielded, using either the average of all images in a given scan or an image taken in the same configuration, but with the FabryPerot removed from the beam. The edges of the illuminated area in each scan were identified by the steepest segment of the intensity dropoff and a circular mask was defined, based on these edge points. The spectral profiles at each position within the masked area were then interpolated onto a $1 \mathrm{~m} \AA$ grid for all subsequent analysis. 
Table 3. List of the FPI scans.

\begin{tabular}{lclccc}
\hline \hline Optical configuration & FPI \# & Date & $t_{\exp }(\mathrm{s})$ & Scan width $(\AA)$ & Illuminated diameter $(\mathrm{mm})$ \\
\hline Collimated & 1 & 2001 Nov. 29 & 0.1 & 0.2 & 30 \\
Collimated & 1 & 2002 Mar. 13 & 0.1 & 0.2 & 30 \\
Collimated & 1 & 2002 Mar. 13 & 0.1 & 0.2 & 30 \\
Collimated & 1 & 2002 Mar. 19 & 1.0 & 0.2 & 30 \\
Collimated & 1 & 2002 Mar. 20 & $0.9,0.2$ & 0.2 & 30 \\
Collimated & 2 & 2002 Apr. 05 & 0.1 & 0.7 & 30 \\
Collimated & 2 & 2002 Jun. 10 & 0.1 & 0.7 & 45 \\
Telecentric & 1 & 2001 Dec. 05 & 0.6 & 0.2 & 45 \\
Telecentric & 2 & 2001 Dec. 05 & 0.6 & 0.7 & 45 \\
Classic & 1 & 2001 Dec. 04 & 0.6 & 0.2 & 45 \\
Classic & 2 & 2001 Dec. 04 & 0.6 & 0.7 & \\
\hline
\end{tabular}

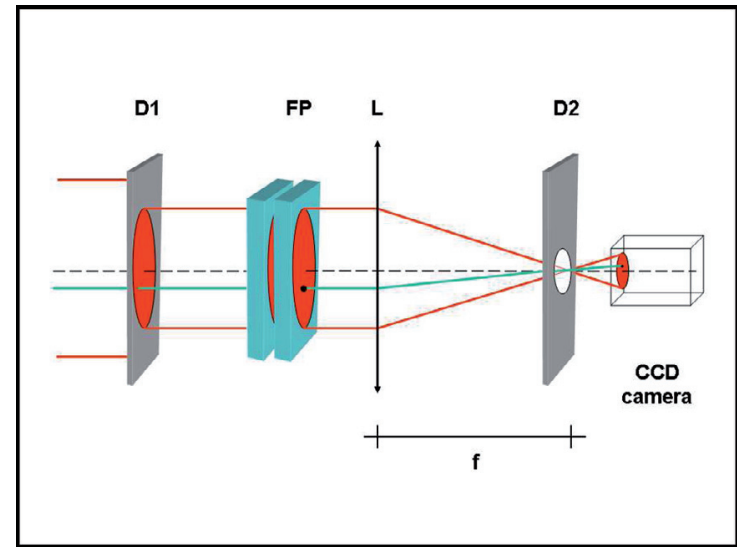

Fig. 1. Schematic layout of the collimated configuration. A frequencystabilized He-Ne laser, a spatial filter and a collimator (not shown in the figure) produce a collimated beam that a first diaphragm (D1) reduces to $30 \mathrm{~mm}$ in diameter. After the Fabry-Perot interferometer, a lens (L) reduces the beam to the dimensions of the CCD camera, where each pixel corresponds to a small area of the interferometer plates, illuminated by a normally incident collimated raybundle (green line). The D2 diaphragm eliminates spurious light due to reflections on the rear surfaces of the interferometer plates.

\section{Collimated configuration}

\subsection{Instrumental layout}

We first measured the interferometer cavity errors using the collimated configuration, shown in Fig. 1. The laser beam was passed through a spatial filter and a collimator, resulting in a beam with a diameter of $\simeq 50 \mathrm{~mm}$. We checked its collimation by means of a shear plate collimation tester, with a typical resolution of $76 \mu \mathrm{rad}$. A diaphragm, $30 \mathrm{~mm}$ in diameter (D1), isolated the more homogeneous inner part of the beam, which was used to directly illuminate the central area of a single FPI, orthogonally to the plates. By means of a lens, placed after the interferometer, the beam was reduced to a size compatible with the dimensions of the CCD, resulting in a final illuminated area with a diameter of $\simeq 900$ pixels. A pinhole, $2 \mathrm{~mm}$ in diameter (D2), was placed at the focus of the lens in order to eliminate spurious images due to reflections on the rear surfaces of the interferometer plates. Images were recorded for each FPI as it was tuned through the wavelengths around the peak of the transmission profile with the minimum voltage step of $1 \mathrm{~V}$.

\subsection{Unresolved cavity defects}

In the collimated configuration, the transparency profile measured at each pixel (hereinafter referred to as pixel profile) directly corresponds to the radiation passing through a finite and unique area of $33 \times 33 \mu \mathrm{m}^{2}$ on the FPI plates, illuminated by a normally incident beam from the laser. If the spacing and the coating changes have a spatial scale sensibly larger than this resolved area, one would expect each pixel profile to have an essentially identical shape as given by the Airy function for an ideal interferometer (without cavity errors) in a collimated beam:

$T=\left(1-\frac{A}{1-R}\right)^{2}\left[1+\frac{4 R}{(1-R)^{2}} \sin ^{2}\left(\frac{2 \pi \mu t \cos \theta}{\lambda}\right)\right]^{-1}$,

where $R$ and $A$ are respectively the coating reflectivity and absorption coefficient, $t$ is the geometrical spacing, $\theta$ the incidence angle, and $\mu$ the refraction index of the material between the plates. For these air-spaced FPI, $\mu$ is nearly unity and essentially constant and in this configuration $\theta$ is 0 . We used the manufacturer's value for the spacing, $t$, which is declared to be accurate to better than $\pm 1 \mu \mathrm{m}$.

However, we found significant variations in the shapes of the observed pixel profiles across the surface of each interferometer. We first quantified these fluctuations by measuring the $F W H M$ $(w)$ at each pixel position within the illuminated field. In Fig. 2 the maps of the measured FWHM are shown, while the relative averaged histograms are plotted in Fig. 3. For both FPI, a large spread in the full-widths was seen, ranging from 0.8 to more

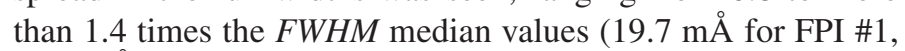
$75.6 \mathrm{~m} \AA$ for FPI \#2). Tests of the effect of photon shot noise or laser intensity fluctuations $( \pm 1 \%$, see Table 1$)$ on the determination of the FWHM showed that the errors due to this noise source are less than $1 \%$ and are not the cause of the broad distribution that is observed.

For an ideal interferometer, we can relate the FWHM of an Airy profile to the coating reflectivity through the following equation:

$\frac{1-R}{\sqrt{R}}=\frac{2 \pi t}{\lambda^{2}} w$

Given the values of the median FWHM found above, the calculated reflectivity for FPI \#1 and FPI \#2 would be, respectively, 0.931 and 0.927 , compared to the nominal value of 0.942 quoted by the manufacturer. Moreover, the broad distribution of $F W H M$ would correspond to reflectivities ranging from 0.945 down to less than 0.90 , a variation significantly larger than expected.

An alternate explanation for the observed fluctuations in the widths of the pixel profiles is unresolved microroughness at 

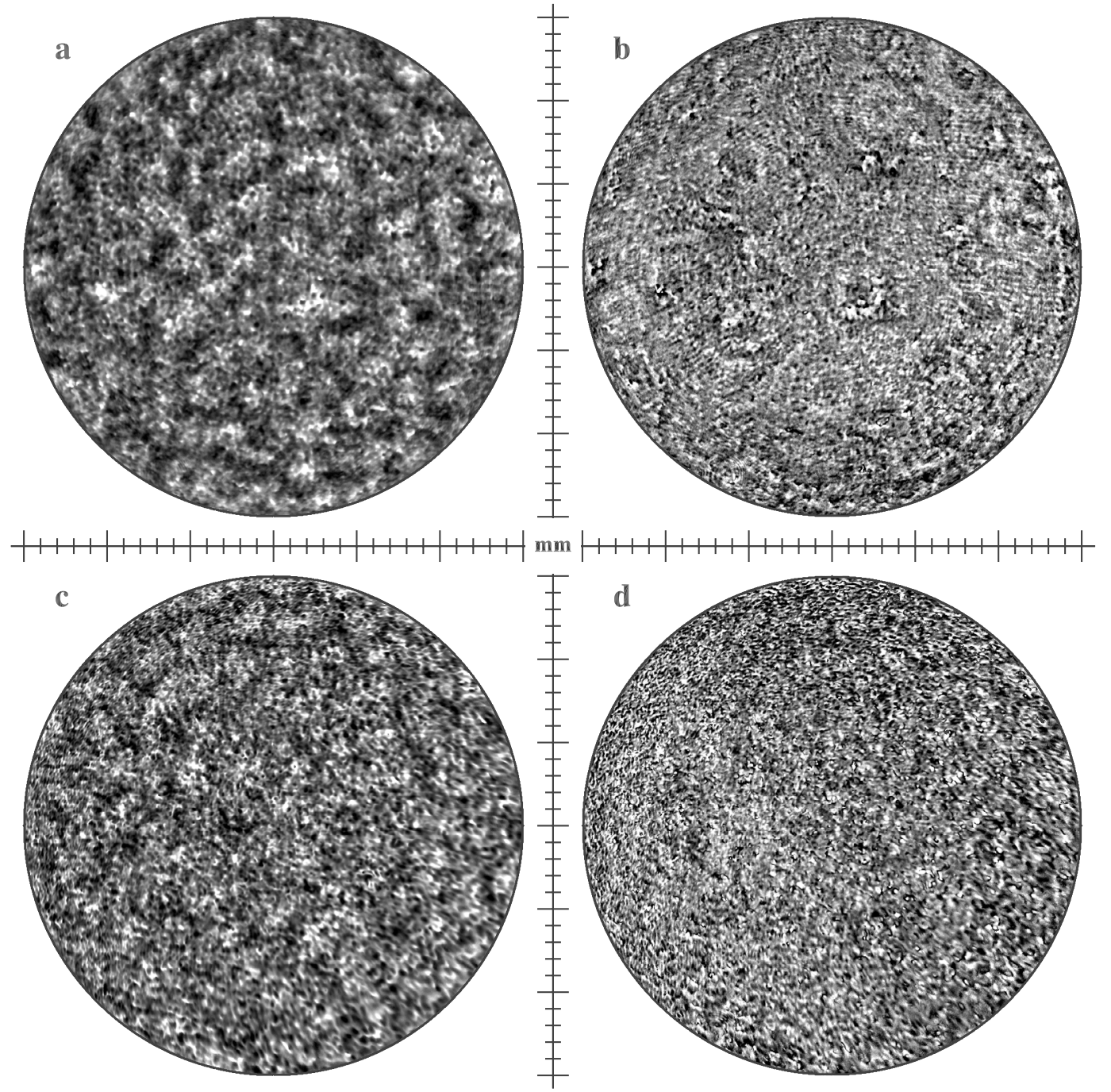

Fig. 2. Maps of the measured FWHM (left column) and profile width ratio (right column) for FPI \#1 $(a, b)$ and FPI \#2 (c,d). The $F W H M$ maps are scaled between 0.8 and 1.4 times the median value of the FWHM for each FPI (see Fig. 3), while the width ratios are scaled between values of 7 and 11 . The tick marks are at a $1 \mathrm{~mm}$ spacing and the maps cover a total diameter of $30 \mathrm{~mm}$.

scales smaller than the $33 \times 33 \mu \mathrm{m}^{2}$ individual elements on the FPI plates. In order to examine how the individual pixel profiles differ from an Airy function, we computed their width ratio, defined as the ratio between the width of the observed profile at intensities of $10 \%$ and $90 \%$ of the transparency peak (Figs. 2 and 4). For an Airy function this width ratio is exactly 9.0, while Fig. 4 shows that a median value of 8.6 was found for both FPI. We hypothesize that the mean shape of the observed pixel profiles for each interferometer is that of an ideal FPI broadened by a Gaussian distribution (which has a lower width ratio of $\sim 4.7$ ) of unresolved microroughness.

In this scenario, the mean pixel profile would be statistically well represented by a Voigt function, resulting from the convolution of an Airy function $T$, for a given reflectivity $R$ (Eq. (2)), and a Gaussian distribution $d_{\mathrm{G}}$ of microroughness errors, defined by a standard deviation $\sigma_{\lambda}$ :

$$
T^{\mathrm{r}}=T(R) * d_{\mathrm{G}}\left(\sigma_{\lambda}\right) .
$$

For each combination of $R$ and $\sigma_{\lambda}$, the resulting profile $T^{\mathrm{r}}$ will have a unique combination of FWHM and width ratio,

The values of $R$ and $\sigma_{\lambda}$ matching the observed FWHM and width ratios were found by constructing synthetic transmission profiles. The expected Airy profile for a given reflectivity was calculated and then we determined the Gaussian distribution which produced a Voigt profile whose FWHM matched the observed median value. The width ratio for this Voigt profile was then measured and the process repeated for a range of values for the reflectivity.

From Fig. 5 we see that the width ratio decreases with increasing reflectivity, because the narrower Airy profile (see Eq. (3)) must be convolved with a broader Gaussian distribution in order to match the observed FWHM. Moreover, the width ratio reaches a limit of 9.0 at that value of the reflectivity for which the Airy profile already has a width equal to the observed value, with no need for additional broadening.

Thus, for the measured width ratio of 8.6, we find from Fig. 5 values of the reflectivity for FPI \#1 and FPI \#2 of 0.935 and 0.931 , which are still significantly below the nominal value of 0.942 given by the manufacturer. The widths of the Gaussian distribution $\sigma_{\lambda}$ are $1.8 \mathrm{~m} \AA$ and $6.4 \mathrm{~m} \AA$, which can be converted from wavelength shifts to spacing fluctuations using Eq. (1), giving $\sigma_{t}^{m}=6.4 \AA$ for both FPI \#1 and FPI \#2, as listed in Table 4 .

We can derive an additional characteristic of the interferometers from this analysis, namely the absorption coefficient 


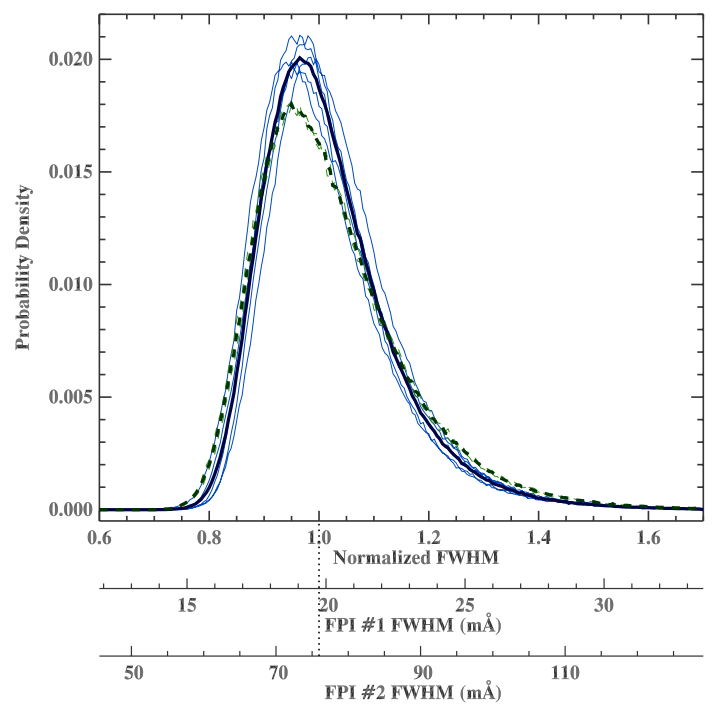

Fig. 3. Averaged histograms of the $F W H M$ measured from the pixel profiles for each interferometer. Solid and dashed lines refer to FPI \#1 and FPI \#2 respectively. The histograms are averaged over all the collimated scans listed in Table 3. Lighter lines are the histograms of the individual scans that went into the averages. Abscissae are plotted both relative to the median FWHM and in terms of the actual profile width for each FPI.

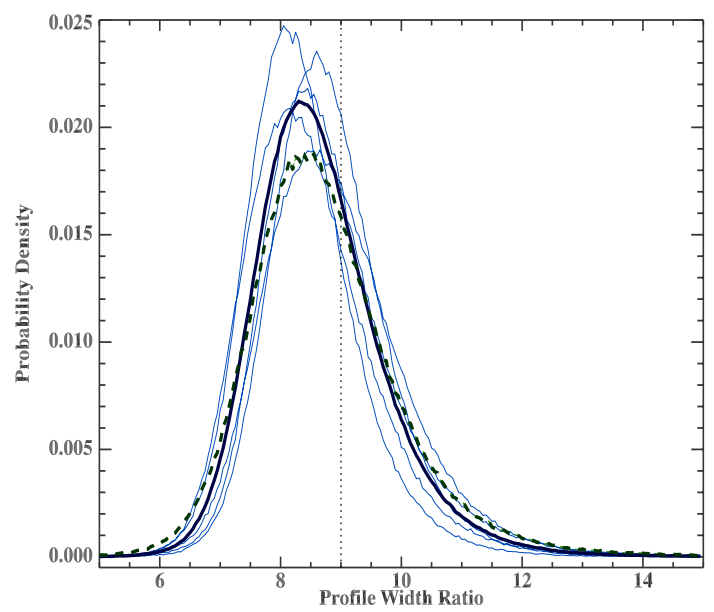

Fig. 4. Averaged histograms of the width ratios measured from the pixel profiles for each of the two interferometers. Solid and dashed lines refer to FPI \#1 and FPI \#2 respectively. Lighter lines show the histograms of the individual scans that went into the averages for each FPI. The vertical dotted line indicates the width ratio expected for an Airy profile.

A (Eq. (2)). Convolving an Airy profile with a Gaussian distribution broadens the resulting Voigt profile while the equivalent width is maintained. So with the increase of the width of the Gaussian distribution, the peak intensity of the resulting profile decreases. Therefore, it is possible to calculate, for the synthesized profiles, the peak intensity for different values of the absorption coefficient.

The peak intensity is plotted versus the width ratio in Fig. 5, for three possible values of $A(0.001,0.002$, and 0.003$)$. Due to the speckles produced by the coherent laser light on the interferometer plates, we cannot calculate a meaningful map of transmission values. However, we can calculate the mean peak transmission over the entire illuminated area, and compare its value with the total illumination over the same area, with the interferometer removed from the path. Taking into account the additional absorption due to the windows $\left(T_{\mathrm{W}}=0.989\right)$ and the
Table 4. FPI cavity errors summary over the central $30 \mathrm{~mm}$ (see Sects. 3.2 and 3.3). All values except the flatness are expressed in $\AA$.

\begin{tabular}{ccccccc}
\hline \hline FPI \# & $\sigma_{t}^{m}$ & $\sigma_{t}^{s}$ & $\sigma_{t}^{r}$ & $\mathrm{P}-\mathrm{V}^{L}$ & Flatness & $\sigma_{t}^{T}$ \\
\hline 1 & 6.4 & 6.1 & 8.8 & 31 & $\lambda / 204$ & 12.1 \\
2 & 6.4 & 6.4 & 9.1 & 27 & $\lambda / 234$ & 10.6 \\
\hline
\end{tabular}

Fabry-Perot plates $\left(T_{P_{\mathrm{FP} 1}}=0.986 ; T_{P_{\mathrm{FP} 2}}=0.983\right)$, as described in Paper I, we find a total transmission of 0.90 and 0.92 for FPI \#1 and FPI \#2, respectively. From Fig. 5 these transparency values, for a measured width ratio of 8.6, correspond to values for $A$ close to 0.002 , consistent with the specification given by the manufacturer (see Table 2).

We also note that for those profiles significantly broader than the median (see Fig. 3), the measured FWHM and peak intensity are both well reproduced by using this model with the value of the reflectivity as determined above, but with anomalously large values of the microroughness (up to $50 \AA$ ).

The adopted model, based on a single Gaussian distribution to describe the unresolved microroughness, is clearly oversimplified. Real microroughness shows a spatial scale dependent behaviour down to sizes of several ångstroms (Elson et al. 1980) which we cannot resolve and which produces strong fluctuations of the shape of the single pixel profiles. In a real instrument, however, the transmission profile for each image point is the result of an integration over a larger area (up to the full interferometer diameter) than for these pixel profiles. We conclude therefore that this proposed model sufficiently describes the mean properties of the plates on a purely statistical basis for the determination of the IBIS transmission profile.

\subsection{Resolved cavity defects}

\subsubsection{Profile shifts}

Much as the statistical properties of the shape of the single pixel profiles have been used to infer the characteristics of spatially unresolved cavity errors, the statistical properties of the wavelength shifts of these same profiles can be used to investigate the spatially resolved spacing defects.

Therefore, we measured the wavelength position of the center of gravity (COG) of each pixel profile $\left(\lambda_{i}\right)$ within the illuminated field. The COG method was chosen because it provides more stable results in the presence of noise and asymmetry in the individual profiles. By subtracting the median $\left(\lambda_{M}\right)$ from the calculated line positions, we obtain a map of relative shifts $\left(\Delta \lambda_{i}=\lambda_{i}-\lambda_{\mathrm{M}}\right)$, which can be finally converted into a map of cavity errors, using Eq. (1). The measured maps for both interferometers are shown on the left side of Fig. 6 where structures on a variety of scales are visible. In the following sections we will individually examine three different components of the observed profile shifts: residual non-parallelism of the plates, large-scale plate defects, and small-scale defects.

\subsubsection{Plate parallelism}

If the angle between the planes fitting the inner surfaces of the two interferometer plates is different from zero, then the interferometer is said to be non-parallel. The resulting gradient in plate separations will introduce a corresponding trend in the profile shifts observed across the aperture of the FPI. The CS100 controller has inputs that can be adjusted in order to change the 

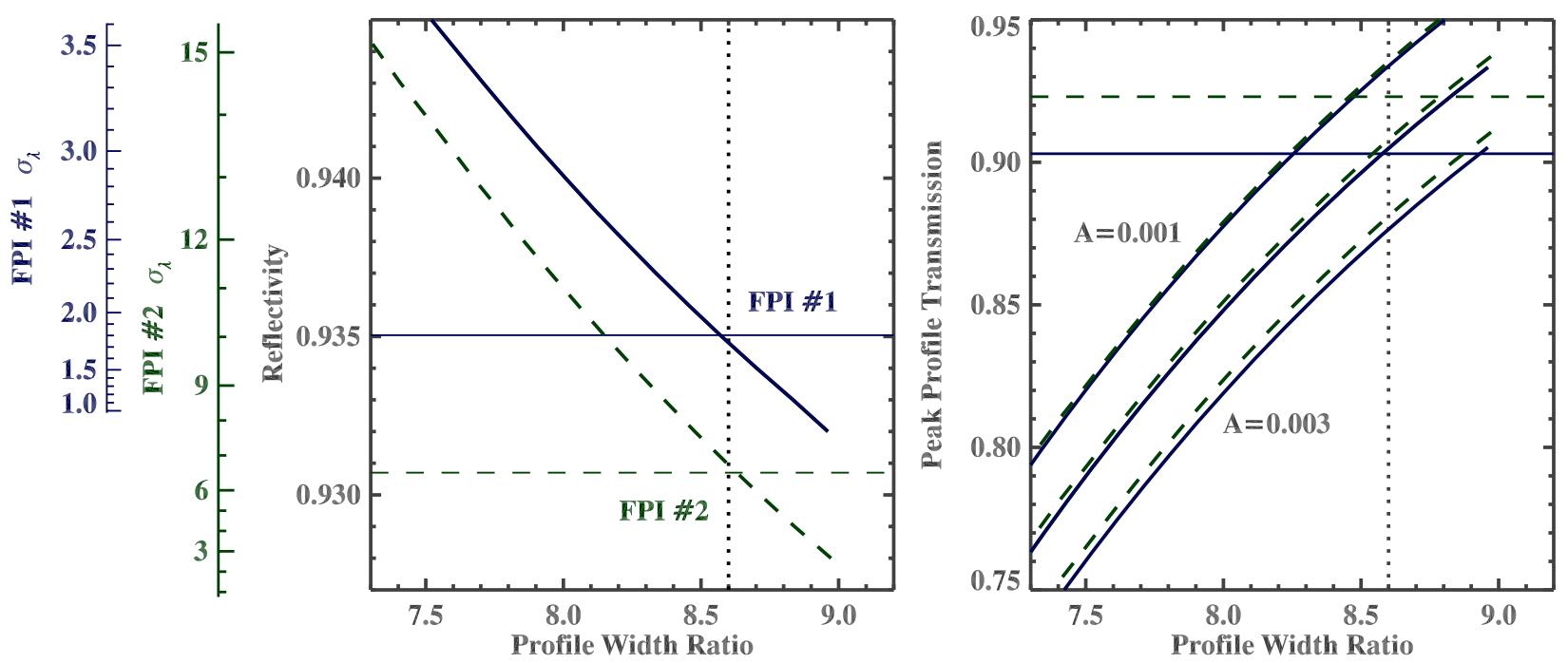

Fig. 5. Model curves calculated from an Airy function $T$ for a given reflectivity (Eq. (2)) convolved with a Gaussian distribution of width $\sigma_{\lambda}$ (mA) such that the $F W H M$ of the resulting profile properly matches the observed median value of the $F W H M$. The width ratio and peak transmission (for three different values of $A$ ) are determined for each convolved profiled. Solid and dashed lines refer to FPI \#1 and FPI \#2 respectively. Left: reflectivity and $\sigma_{\lambda}$ plotted against the width ratio. The vertical dotted line indicates the measured width ratio for both FPI. Right: peak transmission for three different values of the absorption coefficient $A(0.001,0.002,0.003)$ versus width ratio. The vertical dotted line is the same as that on the left, while the two horizontal lines indicate the measured mean peak transmission for FPI \#1 (solid line) and FPI \#2 (dashed line).

relative tilt of the plates, and reference capacitors are used to maintain the plate parallelism. For these tests the adjustments were made manually while observing the pattern of the laser illumination.

Since any residual non-parallelism is not an intrinsic characteristic of the interferometer, it should be removed from the cavity error maps. Therefore, we fitted (and subtracted) a plane to the map of the observed spacing variations separately for each collimated scan (see Table 5, third and fourth column). We find typical angles of 0.01 arcseconds, corresponding to a maximum offset of $20 \AA$ across the plane.

The exact position of the illuminated area on the interferometers varied by several millimeters among the various scans in the collimated configuration (Table 5). As discussed in the following section, the FPI also have significant spacing errors with large spatial scales. The exact distribution of these errors in the illuminated area will influence the fitting of the plane to the measured profile shifts. In order to compare results obtained on different days, we have therefore repeated the fitting using an area common to all measurements performed on each FPI. We find in this case almost identical angles for all the scans (see fifth column in Table 5). For FPI \#1, where we have five measurements spaced over several months, we find mean angles for the $x$ and $y$ directions of $[-0.0116 \operatorname{arcsec},-0.0022 \operatorname{arcsec}] \pm[0.0003 \mathrm{arcsec}$, 0.0006 arcsec]. This result shows that the electronic controller is able to mantain nearly constant parallelism conditions at a distance of several months, which matches our experiences in using IBIS since its installation. We also note that an accuracy of about $\pm 5 \times 10^{-4}$ arcsec can be obtained in measuring the plate parallelism with this procedure.

In principle, once the non-parallelism has been measured, it is possible to physically apply a suitable correction with the interferometer controller, allowing the effective plate spacing to be optimally flattened (Denker \& Tritschler 2005). The presence of large-scale errors (Sect. 3.3.3) implies however that the optimal correction depends on the actual area of the FPI plates used in fitting the plane. We also note, that due to the limited resolution of the CS100, the plate parallelism can be adjusted only within the minimum allowed spacing step (4.8 $\AA$, corresponding to an angular step of $\pm 2 \times 10^{-3}$ arcsec), an accuracy comparable however to the typical microroughness spacing fluctuations.

\subsubsection{Large-scale plate defects}

After the removal of the measured non-parallelism, we are left with a map of the inherent cavity errors of each FPI, showing a large-scale, predominantly radial trend, due to an increasing separation from the center toward the edges of the FPI. To isolate the large-scale errors, we fitted Zernike polynomials up to mode 36 to each observed maps (Denker \& Tritschler 2005).

The resulting distribution is highly asymmetric and we defined a peak-to-valley $(\mathrm{P}-\mathrm{V})$ spacing variation by calculating the range enclosing $99 \%$ of all spacing errors in the fitted surface (thus avoiding the effects of any outlying or anomalous profiles). We obtained a P-V measurement for the large-scale errors, measured over a central $30 \mathrm{~mm}$ aperture, of $31 \AA$ for FPI $\# 1(-11,+20 \AA$ with respect to the median) and $27 \AA$ for FPI \#2 $(-12,+15 \AA)$. The magnitude of the spacing separations can be expressed in relation to the laser wavelength in order to determine a value for the FPI flatness. In this case, the large-scale errors can be expressed as a flatness of about $\lambda / 200$ for both FPI (Table 4).

\subsubsection{Small-scale plate defects}

After removing the fitted Zernike polynomials, only the smallscale spacing errors remain (right side of Fig. 6). We find that these residual spacing errors have an essentially Gaussian distribution with a $\sigma_{t}^{s}$ of $6.1 \AA$ for FPI \#1 and $6.4 \AA$ for FPI \#2 (Table 4). If we assume that these small-scale spacing errors and the previously described microroughness (Sec 3.2) are uncorrelated, we can combine these two Gaussian distributions into a single distribution, accounting for all the essentially randomly distributed errors in the interferometer spacings, with a $\sigma_{t}^{r}$ of $8.8 \AA$ for FPI \#1 and $9.1 \AA$ for FPI \#2 (Table 4). 

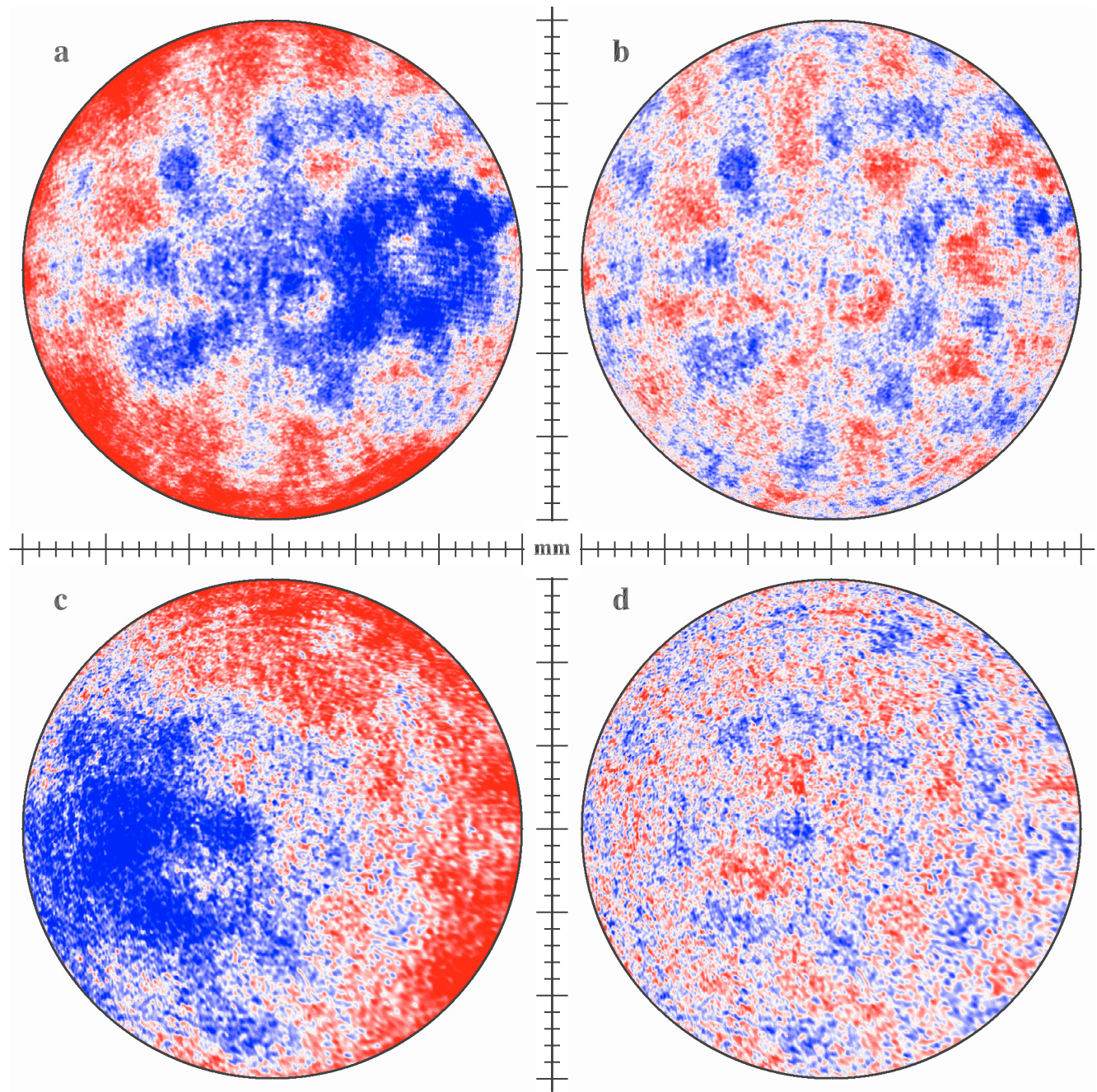

Fig. 6. Maps of the measured wavelength shifts of the pixel profiles (a), c)) and of the same shifts after removal of the plate non-parallelism and the fitted Zernike polynomials (b), d)), for FPI \#1 (top) and FPI \#2 (bottom). For all the images, shifts are scaled between $\pm 25 \AA$ (red positive) of spacing variation, or $\pm 7 \mathrm{~m} \AA$ of profile shifts for FPI \#1 and $\pm 25 \mathrm{~m} \AA$ of profile shifts for FPI \#2.

Table 5. Parallelism measurements.

\begin{tabular}{ccccc}
\hline \hline Scan date & FPI \# & $\begin{array}{c}\text { Fitted plane } \\
\text { P-V }(\AA)\end{array}$ & $\begin{array}{c}\text { Fitted plane } \\
\text { angle }(\operatorname{arcsec})\end{array}$ & $\begin{array}{c}\text { Common area } \\
\text { angle }(\operatorname{arcsec})\end{array}$ \\
\hline 2001 Nov. 29 & 1 & 16.3 & $-0.0110,-0.0023$ & $-0.0119,-0.0032$ \\
2002 Mar. 13 & 1 & 24.7 & $-0.0150,0.0085$ & $-0.0114,-0.0017$ \\
2002 Mar. 13 & 1 & 25.1 & $-0.0160,0.0087$ & $-0.0116,-0.0019$ \\
2002 Mar. 19 & 1 & 22.9 & $-0.0048,-0.0150$ & $-0.0111,-0.0023$ \\
2002 Mar. 20 & 1 & 23.6 & $-0.0058,-0.0150$ & $-0.0117,-0.0020$ \\
2002 Apr. 05 & 2 & 31.7 & $0.0210,0.0072$ & $0.0200,0.0070$ \\
2002 Jun. 10 & 2 & 12.4 & $0.0085,0.0024$ & $0.0130,0.0039$ \\
\hline
\end{tabular}

To verify the correctness of this description, we simulated the situation in which there are no large-scale spacing errors. Hence, the wavelength position of each of the $\simeq 6 \times 10^{5}$ pixel profiles of the field was corrected for the shifts due to the nonparallelism and to the large-scale errors measured above. These corrected profiles were then averaged to obtain a single profile for each FPI that is presumed to be only affected by these small-scale and unresolved cavity errors. This mean profile is compared in Fig. 7 to the Voigt function obtained by convolving an Airy profile (with the reflectance found in Sect. 3.2) and the overall Gaussian distribution of random errors given by $\sigma_{t}^{\mathrm{r}}$ (Eq. (4)). The $F W H M$ of the observed mean profiles are $20.5 \mathrm{~m} \AA$ and $79.0 \mathrm{~m} \AA$ for FPI \#1 and FPI \#2, while the widths of the Voigt function are $20.3 \mathrm{~m} \AA$ and $77.7 \mathrm{~m} \AA$. The differences between the observed and calculated profiles are shown in the lower part of each plot in Fig. 7. For FPI \#1, where five measurements were made, we calculated the rms fluctuation in the normalized intensity at each wavelength position for the five mean profiles. The derived $3 \sigma$ errors bars are plotted in the lower portion of Fig. 7, indicating that these differences are comparable to the measurement errors. 

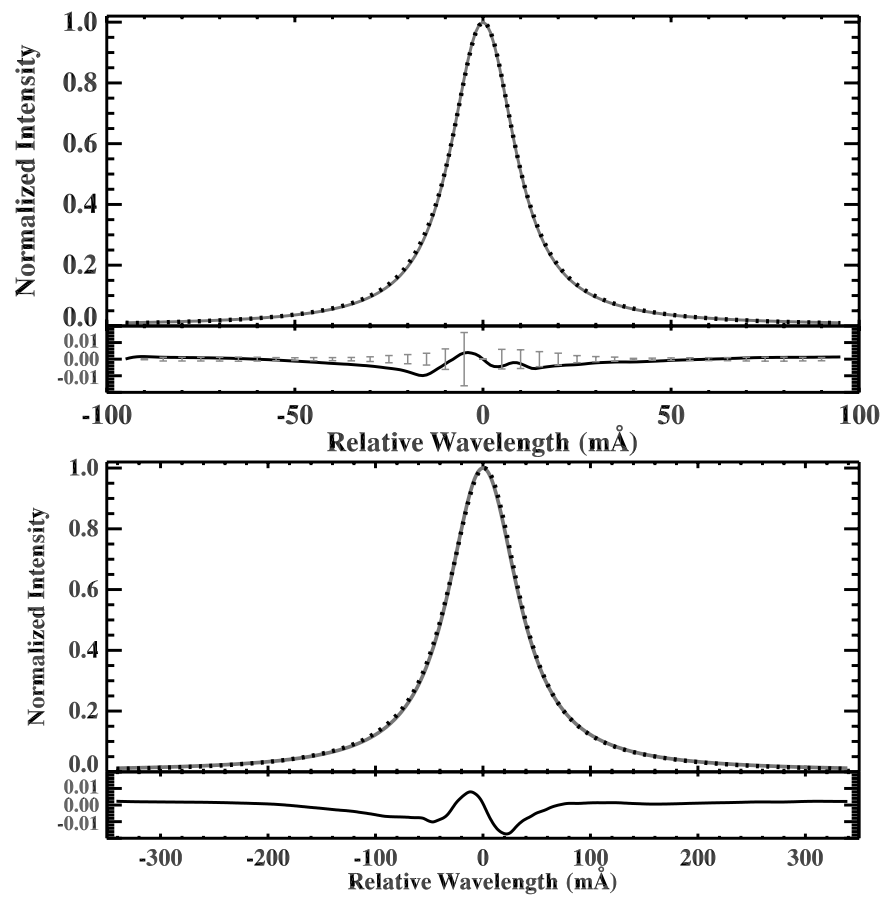

Fig. 7. The mean profiles from the collimated configuration for FP \#1 (top) and FP \#2 (bottom) for an illuminated area of $30 \mathrm{~mm}$. The profiles were averaged after removing only the large-scale errors, leaving the profile broadened by the random spacing variations. The dotted lines shows the convolution of an Airy function defined by the measured interferometer reflectivity (FP \#1: 0.935; FP \#2: 0.931) and a Gaussian function with the measured $\sigma$ (FP \#1: $8.8 \AA$; FP \#2: $9.1 \AA$ ). The lower part of each plot shows the differences between the observed and calculated profiles (including error bars for the measured profile of FP \#1). All the profiles have been normalized to a peak intensity of unity.

However, it could also be argued that these residual differencies are due to a non-rigorous treatment of the effects produced by the cavity errors on the resulting transparency profile. The procedure of convolving an Airy profile with the spacing distribution function is what is generally performed and it is equivalent to considering the total output intensity of the FPI as the incoherent sum of outputs of a distribution of elementary interferometers with different spacings (Vaughan 1989). However, such a procedure is not strictly correct since a more rigorous treatment of the problem should be based on the coherent superposition of amplitudes (Martinez-Herrero et al. 1985; Mahapatra \& Mattoo 1986). In this approach, the wave amplitude is calculated by summing the amplitudes of successively reflected beams, modified by the cavity error distribution, and the output intensity is found by taking its absolute square. In some situations, the result obtained in this manner may be very different from that obtained by convolution. In particular, this procedure can result in a profile whose equivalent width is sensibly reduced due to the cavity defects, while this does not occur with a simple convolution.

We tested this more rigorous approach with our data, but, due to the relatively low amplitude of the cavity errors, the calculated profile was practically identical with the two procedures, with a maximum difference between the profiles on the order of $0.1 \%$. On the basis of this result, we have chosen to use the convolution procedure in all the subsequent analysis.
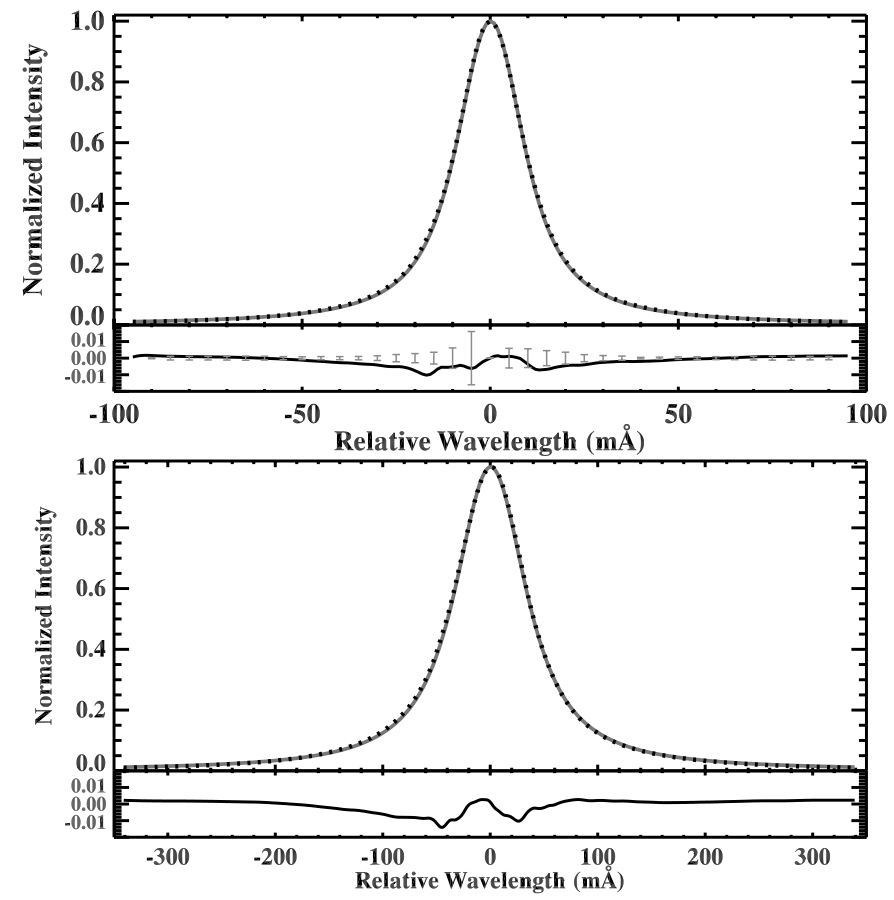

Fig. 8. As Fig. 7, but the profiles were averaged after removing only the plate non-parallelism errors, leaving the profile broadened by all inherent plate spacing variations over the central $30 \mathrm{~mm}$ diameter.

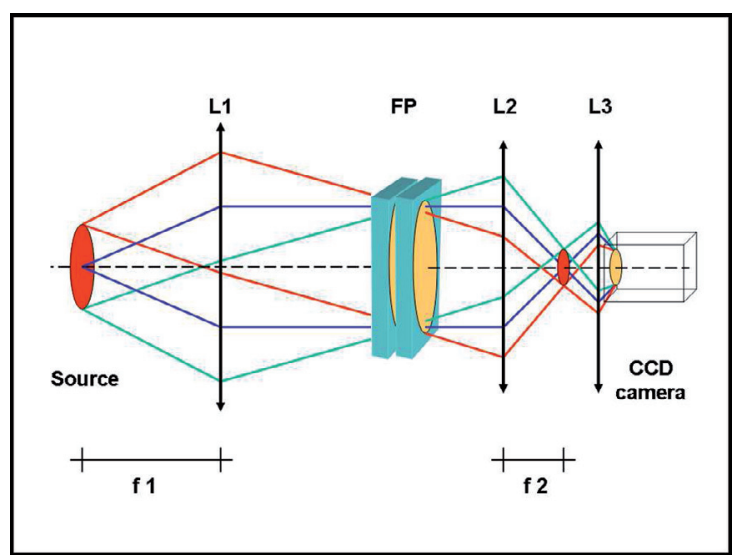

Fig. 9. Schematic layout of the telecentric configuration. A frequencystabilized He-Ne laser (not shown in the figure) illuminates a flashed opal diffuser which simulates a monochromatic incoherent source. The laser light from the diffuser is collimated by a first lens (L1), while two other lenses, after the Fabry-Perot, form an image of the interferometer plates on the CCD camera. In this case, each pixel corresponds to a small area of the plates illuminated by a normally incident cone of rays, containing all the possible directions allowed by the optics.

\subsection{Overall error distribution}

We were thus able to obtain the overall distribution of the intrinsic cavity errors of each FPI by convolving the Gaussian distribution of random errors with the spacing distribution described by the fitted Zernike polynomials. This should provide a complete distribution of the spacing errors at all relevant spatial scales. For the central areas of the interferometers measured in this configuration, the randomly distributed errors on the smaller scales dominate and the resulting distribution still strongly resembles a Gaussian with $\sigma_{t}^{T}=12.1 \AA$ and $10.6 \AA$ for FPI \#1 and FPI \#2, respectively (Table 4). 

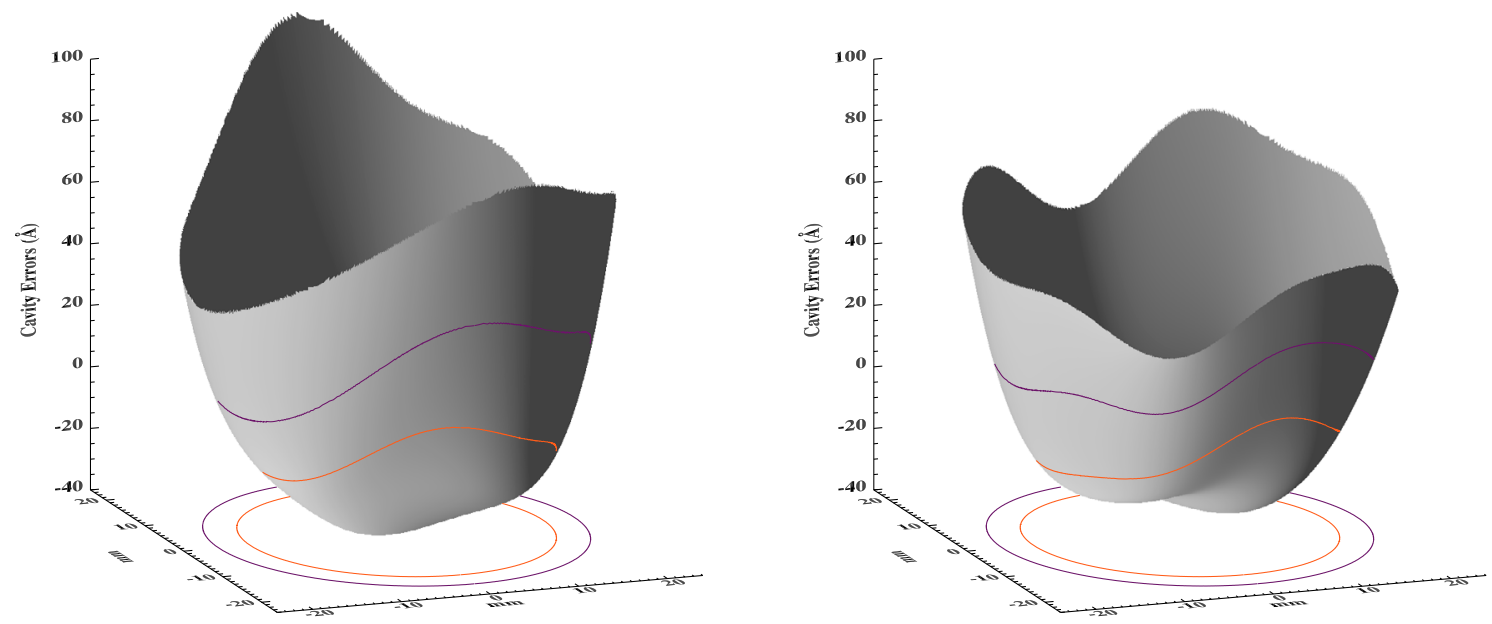

Fig. 10. Surface representation of the Zernike polynomials that were fitted to the cavity errors measured for FP \#1 (left) and FP \#2 (right) in the telecentric configuration. The circles plotted in the $x-y$ plane and the corresponding contours on the surfaces have diameters of $33 \mathrm{~mm}$ (the illuminated area for IBIS) and $40 \mathrm{~mm}$.

Table 6. Coefficients of fitted Zernike polynomials for FPI \#1 and \#2.

\begin{tabular}{lrrlrr}
\hline \hline Mode & $F P I \# 1$ & $F P I \# 2$ & Mode & FPI\#1 & FPI\#2 \\
\hline $\mathbf{1}$ & 44.83 & 9.56 & $\mathbf{1 9}$ & 5.67 & -0.31 \\
$\mathbf{2}$ & -2.22 & -0.87 & $\mathbf{2 0}$ & 0.08 & -1.39 \\
$\mathbf{3}$ & -7.16 & -2.41 & $\mathbf{2 1}$ & -1.76 & 0.70 \\
$\mathbf{4}$ & 72.35 & 31.30 & $\mathbf{2 2}$ & 7.33 & 1.33 \\
$\mathbf{5}$ & -0.79 & -0.90 & $\mathbf{2 3}$ & 0.99 & 1.08 \\
$\mathbf{6}$ & -17.70 & -1.50 & $\mathbf{2 4}$ & -2.20 & 0.20 \\
$\mathbf{7}$ & -7.20 & -3.77 & $\mathbf{2 5}$ & -0.05 & 0.42 \\
$\mathbf{8}$ & -2.30 & -1.49 & $\mathbf{2 6}$ & 1.05 & -0.19 \\
$\mathbf{9}$ & 8.57 & -2.92 & $\mathbf{2 7}$ & -1.71 & 0.62 \\
$\mathbf{1 0}$ & 2.15 & 2.81 & $\mathbf{2 8}$ & -2.42 & 0.44 \\
$\mathbf{1 1}$ & 26.81 & 8.46 & $\mathbf{2 9}$ & -2.00 & -1.37 \\
$\mathbf{1 2}$ & -4.00 & 2.19 & $\mathbf{3 0}$ & 2.00 & -1.20 \\
$\mathbf{1 3}$ & 2.59 & 1.95 & $\mathbf{3 1}$ & 1.67 & -0.51 \\
$\mathbf{1 4}$ & 2.10 & -1.63 & $\mathbf{3 2}$ & 0.11 & 0.71 \\
$\mathbf{1 5}$ & 0.12 & 0.95 & $\mathbf{3 3}$ & -0.80 & -0.42 \\
$\mathbf{1 6}$ & 1.29 & 0.11 & $\mathbf{3 4}$ & -1.44 & 0.60 \\
$\mathbf{1 7}$ & -4.69 & -1.89 & $\mathbf{3 5}$ & 1.20 & -1.08 \\
$\mathbf{1 8}$ & 1.86 & 0.94 & $\mathbf{3 6}$ & 1.03 & -1.14 \\
\hline
\end{tabular}

We again tested the validity of this approach with a similar procedure as in Sect. 3.3.4. The overall observed spacing distribution was convolved with an Airy profile, assuming the reflectivity calculated in Sect. 3.2. We then compared this calculated profile (which is no longer a Voigt profile due to the non-Gaussian distribution of spacing errors) with the measured profile in collimated configuration, after removing any shifts due to plate parallelism errors.

As shown in Fig. 8, once again the calculated transmission profiles very well reproduce the measured mean profiles. The $F W H M$ of the mean profile determined from the observations is $21.8 \mathrm{~m} \AA$ (80.9 $\mathrm{m} \AA$ ) for FPI \#1 (FPI \#2), while the width of the convolved profile is $21.6 \mathrm{~m} \AA(79.8 \mathrm{~m} \AA)$.

\section{Telecentric configuration}

Because of the limits of the collimator used to enlarge the laser beam in the collimated configuration described above, we were only able to quantify the cavity spacing defects over an area of $30 \mathrm{~mm}$. In order to evaluate the instrumental profile for larger areas, we made an additional set of scans in a second

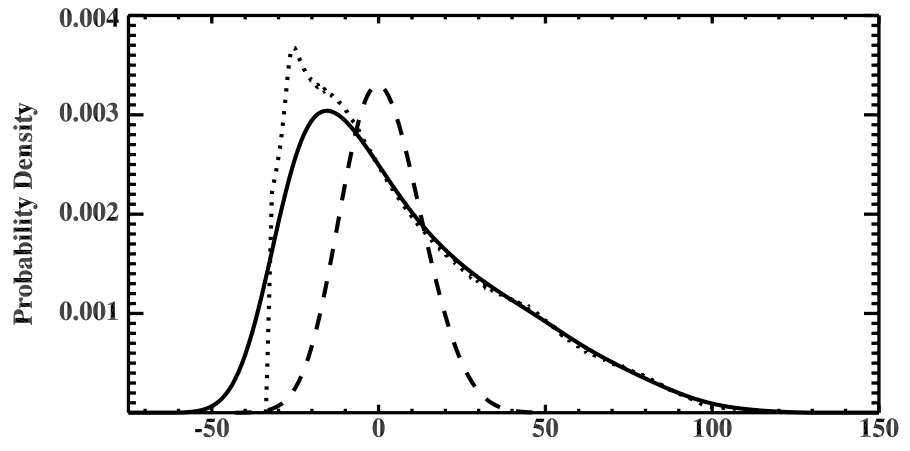

Fig. 11. Histogram of plate spacing fluctuations $(\AA)$ for FP \#1 over the central $45 \mathrm{~mm}$ of the interferometer (solid line), as determined by convolving the distribution of separations in the fitted Zernike polynomials (dotted line) with a Gaussian distribution with a width $\sigma_{t}^{r}$ as given in Table 4. For comparison, the similarly derived distribution for a $30 \mathrm{~mm}$ aperture (see Sect. 3.4) is also shown (dashed line).

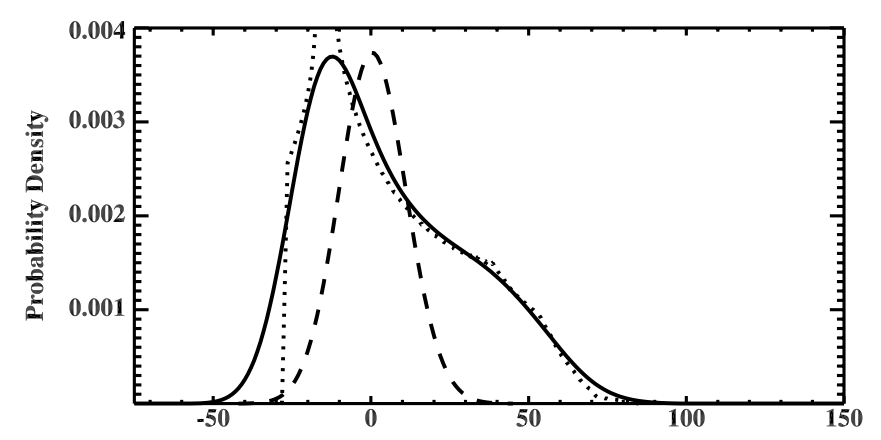

Fig. 12. As Fig. 11 for FP \#2.

configuration, the telecentric layout (Fig. 9), that allows the profile shifts to be measured over nearly the full aperture of the interferometer.

In this layout, the laser beam passes through a circular rotating diffuser near its outer edge where it forms a small bright spot with a rapidly changing speckle pattern. This spot illuminates a second flashed opal diffuser a short distance away, which simulates an extended monochromatic and incoherent source at the focus of the first lens (L1). Two other lenses, after the FPI, form an image of the interferometer plates on the CCD camera. As 


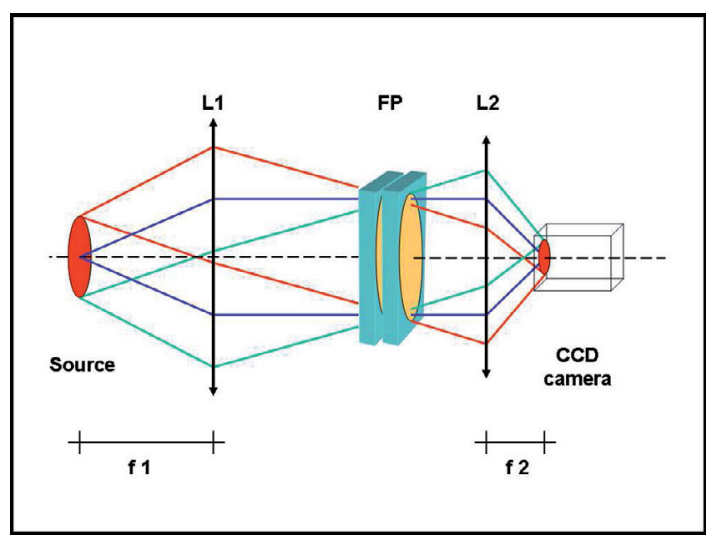

Fig. 13. Schematic layout of the classic configuration. The layout is similar to that of Fig. 9, but with the CCD camera at the focus of the lens L2, where an image of the diffuser is formed. In this case, each pixel corresponds to a collimated beam incident with a given angle on the interferometer plates.

shown in Fig. 9, the optics simulates a telecentric configuration where each image point is formed by an equal cone of rays normally incident on the plates. A diaphragm placed in front of each interferometer limited the illuminated area to a circle $45 \mathrm{~mm}$ in diameter.

As in collimated configuration, each pixel in the resulting image corresponds to a small distinct area $\left(\simeq 50 \times 50 \mu \mathrm{m}^{2}\right)$ on the interferometer plates, but in this case, each pixel profile is broadened due to the finite aperture $\left(0.57^{\circ}\right)$ of the incident cone of rays and is far from a simple Voigt function. This configuration does not therefore allow for the precise quantification of the microroughness as with the collimated layout. However, since the microroughness is a result of the polishing and coating processes and we observe it to be fairly constant over the entire substrate, we will assume that the distribution of random errors (characterized by $\sigma_{t}^{T}$ ) measured over the central $30 \mathrm{~mm}$ of each FPI is valid for the full aperture of both interferometers.

In the telecentric layout, the shifts in wavelength of the profiles depend on the spacing defects of the individual area corresponding to that profile. Thus we used the center-of-gravity method to quantify the shifts and Eq. (1) to convert them to spacing defects, resulting in maps of the resolved plate errors over the full illuminated area. We removed a plane fitting the non-parallelism errors and, as in Sect. 3.3.3, we fitted the residual plate defects with Zernike polynomials up to mode 36 . The coefficients of the fit are listed in Table 6 and the complete fitted polynomials are shown in Fig. 10. Both surfaces show a strong radial trend (as can also be seen in the magnitude of the fourth Zernike mode, a parabola), with the plate separation increasing toward the edge of the plates. This apparent "bowing" could be due to errors or deformation of the FPI plates themselves or to the stresses in the multi-layer coating applied to their surface.

The fitted polynomials were used to generate a histogram of the spacing errors of each interferometer (dotted lines in Figs. 11 and 12), which was then convolved with the Gaussian distribution of the small-scale plate defects derived in Sect. 3.3.4. The resulting distributions (solid lines in Figs. 11 and 12) should represent the full range of cavity errors at all spatial scales over the measured $45 \mathrm{~mm}$ aperture of each FPI.

Over the full aperture of the FPI, the large-scale errors essentially determine the overall distribution of the plate spacings. The peak-to-valley values of this distribution over the $45 \mathrm{~mm}$ diameter are approximately four times larger than those found on
Table 7. FPI cavity errors summary over the central $45 \mathrm{~mm}$ (see Sect. 4).

\begin{tabular}{cccc}
\hline \hline FPI \# & $\sigma_{t}^{r}$ & $\mathrm{P}-\mathrm{V}^{L}$ & Flatness \\
\hline 1 & 8.8 & $-32,+89$ & $\lambda / 52$ \\
2 & 9.1 & $-27,+67$ & $\lambda / 67$ \\
\hline
\end{tabular}
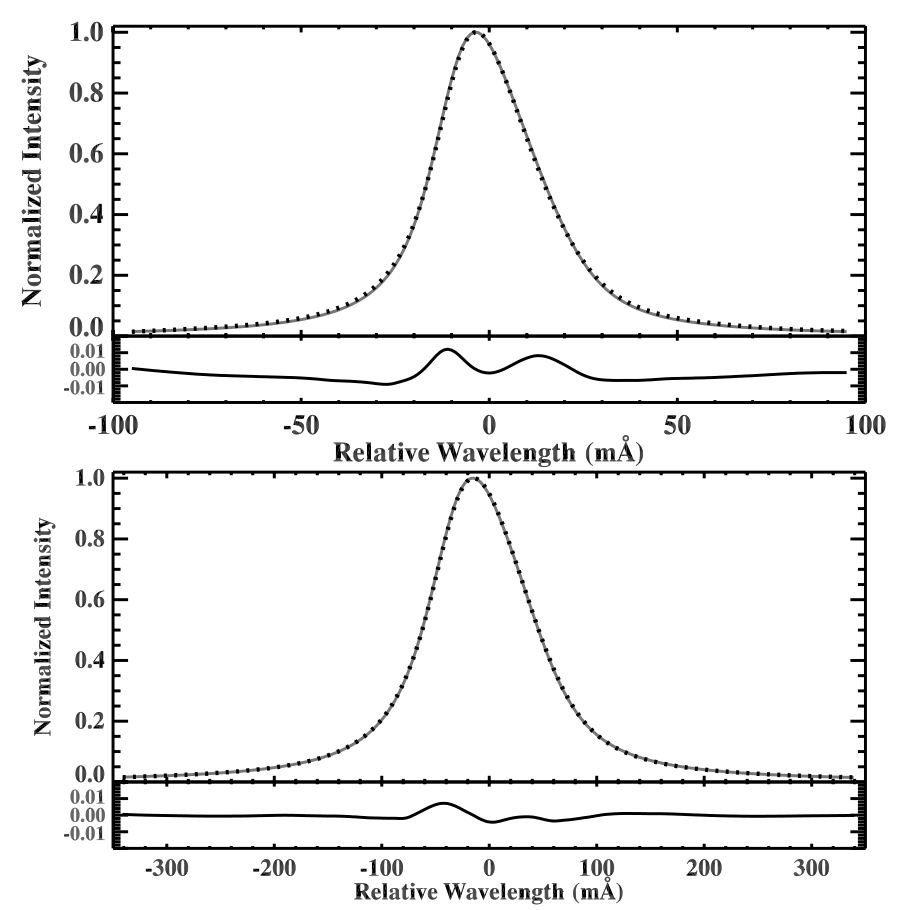

Fig. 14. The mean profiles from the classic configuration for FPI \#1 (top) and FPI \#2 (bottom). The profiles were averaged after removing the instrumental blue-shift, leaving them broadened by all inherent plate spacing variations. The dotted line shows the convolution of an Airy function defined by the derived interferometer reflectivity (FPI \#1: 0.935; FPI \#2: 0.931) and the full distribution of spacing errors measured on a $45 \mathrm{~mm}$ aperture in telecentric configuration. The lower part of each plot shows the differences between the measured and calculated profiles. All the profiles were normalized to a peak intensity of unity.

the smaller area $(30 \mathrm{~mm})$, with a reduction of the plate flatness to about $\lambda / 50$ (see Table 7).

\section{Classic configuration}

To verify that the plate spacing histograms derived in the previous section are correct, we used a third layout, similar to the telecentric configuration, but with the CCD camera in the focal plane of the second lens. As shown in Fig. 13, this setup simulates a classic mount where an image of the diffuser is formed on the camera and each point in the image is formed by identical collimated beams that are incident on the interferometer plates at different angles. The same diaphragm was used as in the telecentric configuration, resulting in the same $45 \mathrm{~mm}$ illuminated area.

In this case, since each beam illuminates the same area of the FPI, the resulting transparency profiles are all the result of an integration over the same distribution of shifts due to the cavity defects and should therefore have the same shape, while their wavelength position shows a parabolic blue-shift towards the edge of the field due to the increasing angle of incidence. The 
profiles measured at each pixel were shifted to remove the relative wavelength shifts and averaged to reduce noise.

We compare for each FPI this averaged profile to the transparency profile calculated by convolving the Airy function (defined by the derived coating reflectivity from Sect. 3.2) with the relative overall distribution of errors derived in the previous section. Once again, as shown in Fig. 14, close agreement is found between the observed and calculated profiles, indicating the correctness of the measured overall error distribution and demonstrating that the adopted procedure is suitable for generating the transmission profile for any given area of each interferometer.

\section{Instrumental profiles}

\subsection{IBIS instrumental profile at $6328 \AA$}

Having characterized in depth each of the interferometers individually, we now examine the case when two FPI are used in series, as in the IBIS instrument. For ideal interferometers the overall transmission profile is simply the product of the transmission profiles of the two FPI. For real interferometers, however, it is important to take into account the effects of the surface defects.

In actual use, the operation of two FPI in series requires a tuning process, in which the spacing of each interferometer is adjusted to have, at a desired wavelength, an optimum alignment between the transmission profiles of the two FPI resulting in a maximum overall integrated transmission. While this produces a best average correspondence between the two transmission profiles, the different spacing fluctuations in individual elemental areas traversed by a beam illuminating the two interferometers will result in relative shifts in the FPI transmission profiles. The situation is equivalent to a local detuning, which produces a broadening of the resulting transparency profile and a loss of the overall transmittance (Paper I) and has been named mutual masking (Mack et al. 1963).

If the spacing errors are randomly distributed and uncorrelated between the two FPI, it can be shown that the resulting profile is simply the product of the two interferometer profiles, each broadened by the suitable distribution of wavelength shifts arising from the surface defects. In the case of large-scale spacing fluctuations, such as those measured above, the calculation is instead more complicated, since the error distributions are no longer symmetric nor completely uncorrelated. This requires that we compute the overall profile $T_{\mathrm{o}}$ as a mean of the products of the two transmission profiles calculated for each point in the field of view, written as

$T_{\mathrm{o}}(\lambda)=\frac{1}{n^{2}} \sum_{x, y=1}^{n} T_{1}^{\mathrm{r}}\left(\lambda+\Delta \lambda_{1}(x, y)+s_{1}\right) T_{2}^{\mathrm{r}}\left(\lambda+\Delta \lambda_{2}(x, y)+s_{2}\right)$

where $(x, y)$ are all the positions in the illuminated area, $T_{i}^{\mathrm{r}}(\lambda)$ is the FPI profile broadened by the roughness distribution (Eq. (4)), $\Delta \lambda_{i}(x)$ is the wavelength shift due to the large-scale plate defects at position $(x, y)$ for each of the FPI, and $s_{i}$ are the optimum offsets of each FPI as determined from the tuning process.

Because of presence of the large-scale cavity errors, the result of the tuning procedure will generally depend on the areas of the FPI actually utilized. Therefore, we performed a synthetic tuning by calculating the instrumental profile for the selected interferometer area for a range of values $s_{1}$ (setting $s_{2}=0$ ) and finding the offset for which the total transmittance through the two FPI is maximized.

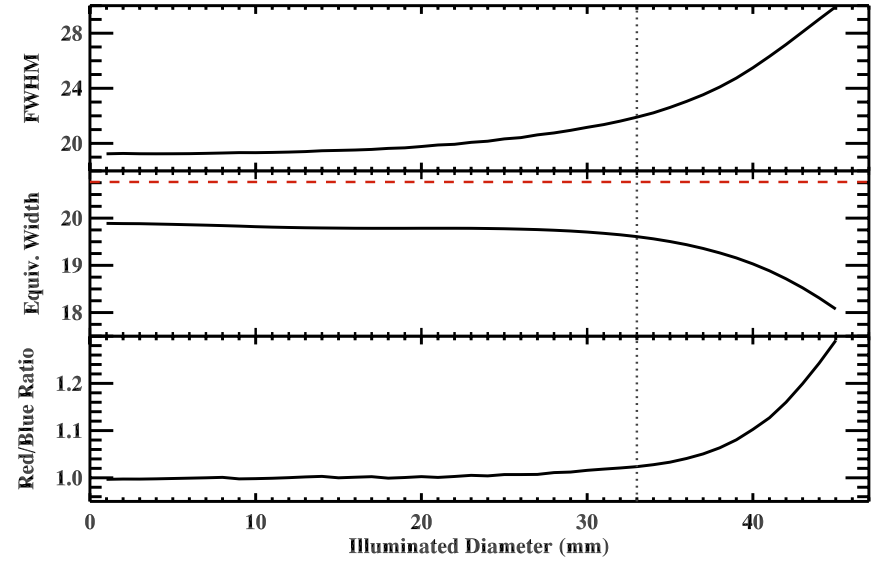

Fig. 15. Plots showing the variations of several parameters of the instrumental profile at the $6328 \AA$ as a function of the total illuminated diameter on the interferometers in a dual FPI system using a classic mount. The $F W H M$ and equivalent width are given in $\mathrm{m} \AA$. The profile ratio is calculated from the relative contribution of two halves of the profile on either side of the peak intensity (see Eq. (6)). The vertical dotted line indicates the illuminated diameter for IBIS and the dashed line in the middle panel shows the equivalent width of the combined profile in the case that the FPI were absent of any plate defects.

Using this procedure, we calculated the overall instrumental profile at the laser wavelength in a range of $\pm 1 \AA$ around the central peak for areas of increasing diameter. We then determined the FWHM and equivalent widths (normalized to the total illuminated area) for these profiles (Fig. 15). Over the central areas of the FPI, within a diameter of approximately $30 \mathrm{~mm}$, the distributions of large-scale spacing errors are mostly symmetric and comparable to the small-scale random errors. This results in an instrumental profile that varies little for diameters within this range. However, as the diameter increases, the distributions of the spacing errors become broader and increasingly asymmetric, causing the width of the resulting profile to grow rapidly. At the same time, the increasing mutual masking results in a decrease in the equivalent width of the transmission profile and hence a reduced instrumental transmittance. This decrease, with respect to the profile calculated in the absence of any plate defects, is approximately $5 \%$ for diameters up to $30 \mathrm{~mm}$, but rapidly increases up to $13 \%$ for the $45 \mathrm{~mm}$ aperture.

The asymmetry of the large-scale error distribution also produces an asymmetry in the instrumental profile itself. We quantify this effect by calculating the ratio

$a=\int_{\lambda_{0}}^{\lambda_{r}} T_{\mathrm{o}} / \int_{\lambda_{b}}^{\lambda_{0}} T_{\mathrm{o}}$

where $\lambda_{0}$ is the wavelength of the peak of the transmission profile and $\lambda_{b}$ and $\lambda_{r}$ are, respectively, the blue and red wavelength limits of the calculated profile. Again, within a diameter of about $30 \mathrm{~mm}$, the contributions from the two halves of the profile are essentially equal, but with an increasing illuminated diameter, the profile becomes more weighted to the red (bottom panel of Fig. 15).

We tested this method for calculating the overall instrumental profile for two interferometers used in series by comparison with the observed transmission through FPI \#1 and FPI \#2 as measured on 14 November 2007 with IBIS using the laser calibration channel (see Paper I). We used a classic mount configuration, as described in Sect. 5, to illuminate a $35 \mathrm{~mm}$ aperture on 


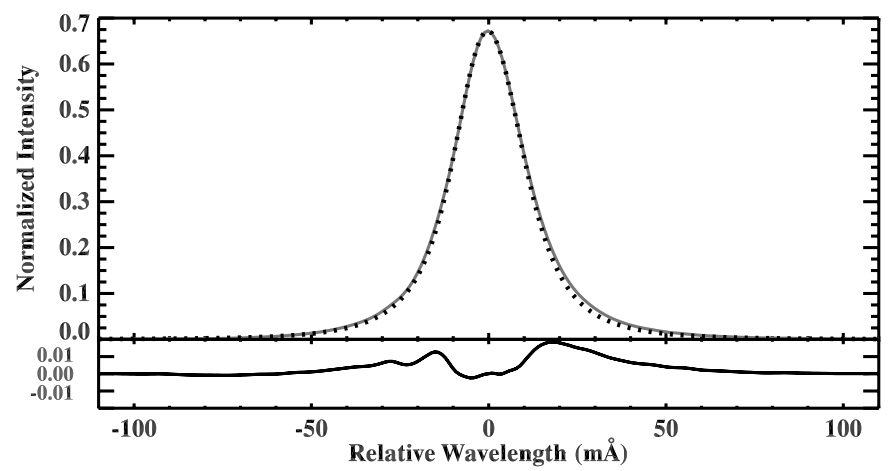

Fig. 16. The mean profile (solid line) from the measurement of the transmission profile through FPI \#1 and FPI \#2 in series. The profile, averaged after removing the instrumental blue-shift, was measured over an area of $35 \mathrm{~mm}$ on the interferometers. The dotted line shows the calculated multi-etalon profile using Eq. (5), while the lower plot shows the difference between the measured and calculated profiles. The observed profile has been normalized to the peak intensity of the calculated profile.

both FPI. The two interferometers were both accurately aligned orthogonal to the collimated laser beam and a neutral density filter was placed between the two FPI to eliminate any ghosts due to inter-reflections. The transmission profiles over the central area of the image were shifted to correct for the field dependent blueshift and averaged to get the mean profile shown in Fig. 16. The FWHM of the measured transmission profile is $24.0 \mathrm{~m} \AA$, while the with of the profile calculated using Eq. (5) is $23.4 \mathrm{~m} \AA$. The two profiles are quite similar, though the wings of the observed profile are slightly higher than those of the calculated profile. This may simply be a result of the difficulty in achieving an accurate alignment of the optical layout with the instrument installed at the telescope rather than in the laboratory, or it may indicate the effects of aging on the interferometer coatings in the six years since the original laboratory measurements.

\subsection{IBIS instrumental profile vs. wavelength}

The above measurements and calculations were performed at the wavelength of the He-Ne laser, but more generally we are interested in the instrumental profile at the wavelengths within the IBIS operative range (5800-8600 ̊).

The wavelength dependence of the shifts due to the cavity errors can be easily calculated by Eq. (1), as the spacing fluctuations have been quantified in terms of an effective variation of the plate spacing.

The reflectivity of the interferometer plate coating also varies with wavelength, as documented by the FPI manufacturer with the curve shown in Fig. 17. However, since we find at the laser wavelength an effective reflectivity lower than that quoted by the manufacturer, we must scale this curve at all wavelengths so as to match the measured value. We choose to use an additive correction under the hypothesis that the lower effective reflectivity is due to an additional loss or scattering not accounted in the measurement of the coating transmission (the results with a multiplicative correction would be essentially identical). The adjusted curves for FPI \#1 and \#2 are also shown in Fig. 17.

The instrumental profile was calculated at wavelength steps of $100 \AA$, from 5400 to $9000 \AA$, using the same procedure as for the laser wavelength. At each wavelength a separate synthetic tuning was performed to find the plate spacings providing the maximum overall transmittance. The profiles were calculated for

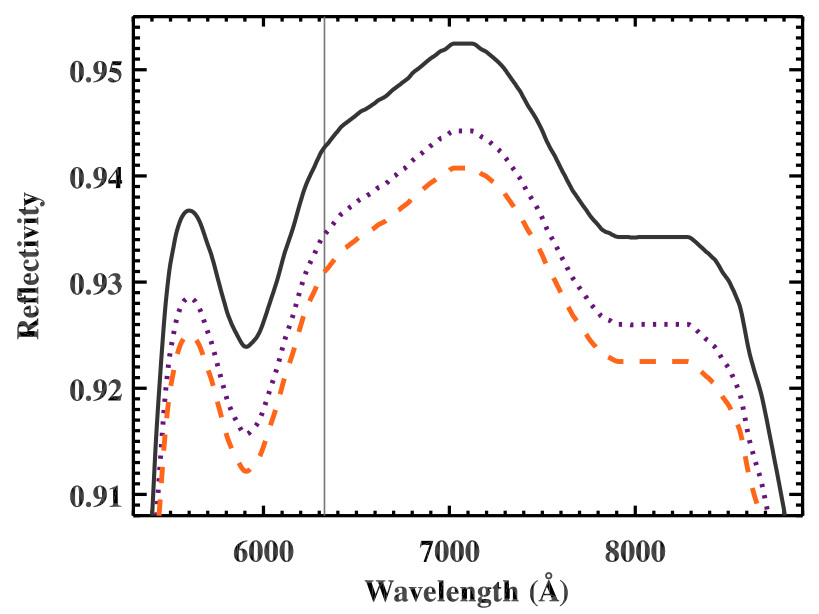

Fig. 17. The coating reflectivity of the interferometer plates as a function of wavelength. The solid line shows the reflectivity curve provided by the manufacturer, while the dotted and dashed line show this curve for FPI \#1 and FPI \#2 respectively, after being shifted to match the values of the reflectivity found at the laser wavelength (vertical line).

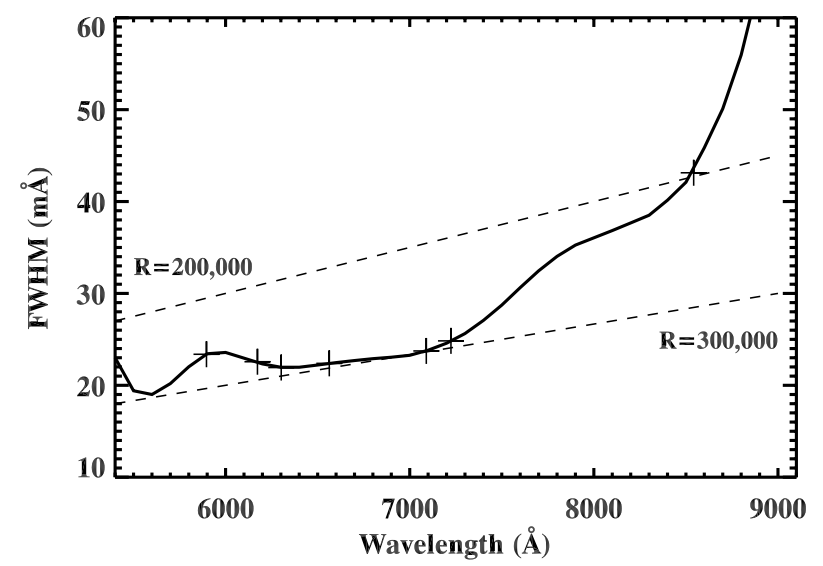

Fig. 18. The $F W H M$ of the IBIS instrumental profile vs. wavelength. The crosses indicate the wavelengths of several currently available interference filters (Paper I). The diagonal dashed lines show contours of constant spectral resolving power $(\lambda / F W H M)$, indicating IBIS achieves a resolution of close to 300000 over most of its wavelengths range below $7300 \AA$.

a range of $\pm 25 \AA$ around the central peak and using the central $33 \mathrm{~mm}$ diameter of the FPI, which corresponds to the diameter of the telescope pupil for IBIS as installed at the DST.

In Fig. 18, we plot the FWHM of the instrumental profile, which depends in part on the wavelength variation of the coating reflectivity. We note that the spectral resolving power remains close to 300000 over the lower half of the instrument wavelength range and only dips below 200000 beyond $8500 \AA$.

A further quantity often used to characterized the spectral performance of FPI-based instruments is the level of the parasitic light, defined as the percentage of the overall transmittance that comes from outside the two closest minima on either side of the central transparency peak. In order to evaluate this quantity, we must apply a transparency profile for the prefilter that isolates the central peak (Paper I). Using the theoretical transmission profile for a two-cavity prefilter with a $F W H M$ of either 3 and $5 \AA$, we derive the level of parasitic light over the full range of wavelengths. As can be seen in Fig. 19 the narrower filter is necessary at the shorter wavelengths in order to maintain the parasitic light at less than $2.5 \%$ of the total transmittance. 
At longer wavelengths, above approximately $7000 \AA$, a prefilter with a $F W H M$ of $5 \AA$ is sufficient to maintain the parasitic light under $1.5 \%$.

We also include in our calculation the computation of the ghost profile due to inter-reflections between the the two FPI in an orientation normal to the optical axis, as described in Paper I. We find that in the configuration used for IBIS, placing an interference filter with a transmission of $30 \%$ between the two FPI, the equivalent width of the ghost profile is approximately $1 \%$ of that of the primary transmission profile at all wavelengths.

From the observational point of view, it is perhaps more meaningful to measure the wavelength limits which enclose some specified percentage of the total instrumental transmittance. In Fig. 20 the wavelength range which encloses $95 \%$ of the total transmission has been plotted. The dominant contribution to observed intensity comes from within a range of $100-150 \mathrm{~m} \AA$ around the central peak, averaging approximately 3.7 times the $F W H M$. This curve is plotted for prefilters with both a $3 \AA$ and $5 \AA F W H M$ and it is even more clear that the narrower prefilter is required for the shorter wavelengths, while above $7000 \AA$ the wider prefilter can be used.

\subsection{Comparison to designed IBIS characteristics}

From all of the above results it is clear that the design of an FPIbased instrument needs to take into account the magnitude and distribution of the cavity errors on different scales as they play an important role in determining the overall system performance. During the design of IBIS, the characteristics of the FPI, still to be acquired, were not available. The manufacturer claimed the large-scale errors to be on the order of $\lambda / 150$ after coating. A more conservative value of $\lambda / 100$ was assumed (Paper I) and, following Ramsay (1969) and Netterfield \& Ramsay (1974), these errors were taken to be due to a symmetric parabolic nonuniformity. By limiting the illuminated area of both FPI to less than $35 \mathrm{~mm}$ in diameter, the further design was based on a presumed rectangular error distribution with a $F W H M=\lambda / 200$.

Our measurements confirm that the large-scale errors have a largely parabolic shape, as shown by the predominance of the of the Zernike mode 4 coefficient (Table 6), but for the $33 \mathrm{~mm}$ area utilized in IBIS the small-scale roughness defects are also significant. The overall result are Gaussian-like error distributions with widths of $\lambda / 204$ and $\lambda / 234$ for FPI \#1 and FPI \#2 (see Table 4).

The hypothetical and the measured cavity error distributions (for the utilized $33 \mathrm{~mm}$ diameter) have different shapes but a comparable FWHM. Because neither distribution greatly broadens the Airy function for these FPI in the absence of plate defects, the spectral resolving power is similar in both cases: $200000-270000$ in Paper I and 200000-300000 determined in this work. The higher maximum value we find (300000 versus 270000) is due to the higher reflectivity of the interferometer coating (even after applying the correction determined in Sect. 3.2) in some portions of wavelength range (Fig. 17), while in Paper I a constant value of 0.930 was assumed for the reflectivity.

The IBIS design also attempted to maximize the spectral purity of the observed line profiles. We find that the parasitic light remains below the $2.5 \%$ design specification given in Paper I and actually remains below $1.5 \%$ over most of the spectral range. The calculations also indicate that this level of spurious light can be maintained for interference filters with $F W H M$ of $5 \AA$ down to wavelengths of $7000 \AA$ (compared to the limit of $7500 \AA$ given

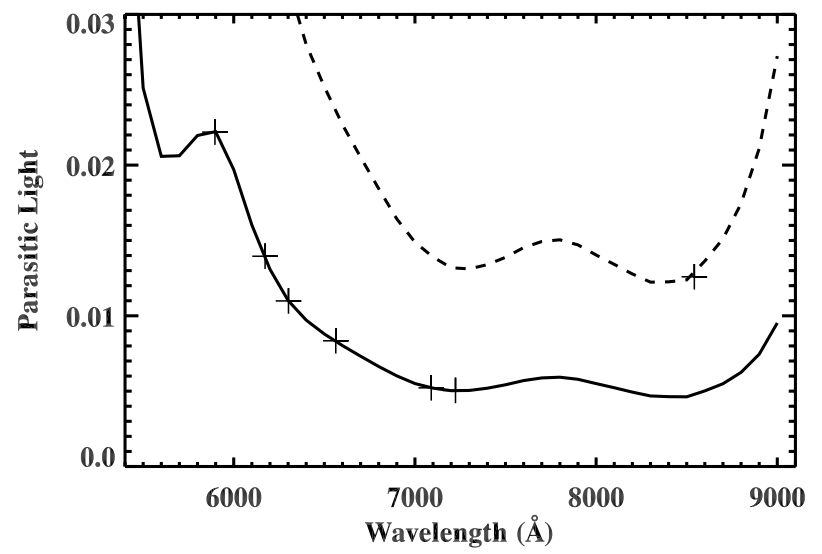

Fig. 19. The parasitic light of the IBIS instrumental profile vs. wavelength. This quantity has been calculated by supposing in series with the two FPI a theoretical two-cavity interference filter, with a FWHM of $3 \AA$ (solid line) and $5 \AA$ (dashed line). Crosses indicate the wavelengths of several currently available interference filters for IBIS.

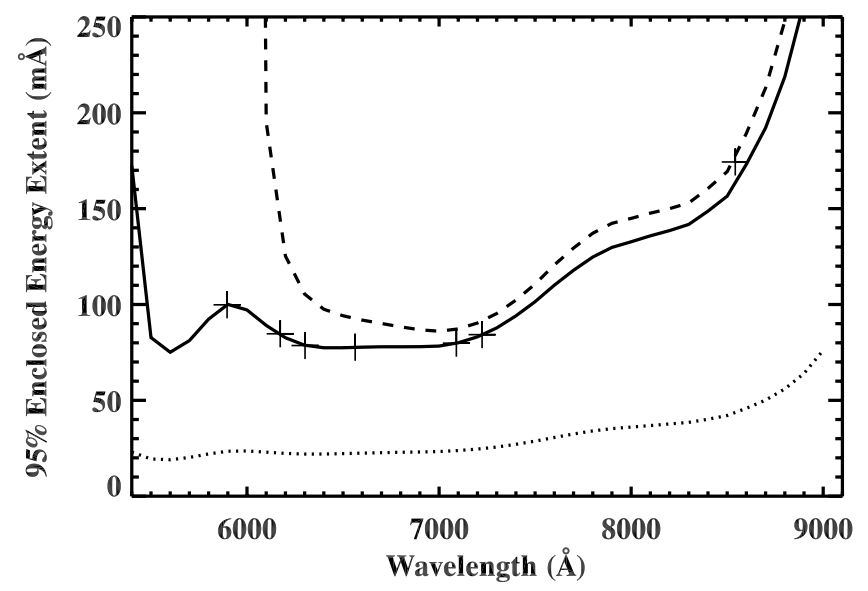

Fig. 20. The wavelength range around the central transparency peak which encloses $95 \%$ of the entire energy transmitted by the instrumental profile, vs. wavelength. The enclosed energy range was calculated by supposing in series with the two FPI a theoretical two-cavity interference filter, with a $F W H M$ of $3 \AA$ (solid line) and $5 \AA$ (dashed line). Crosses indicate the wavelengths of several currently available interference filters for IBIS. The dotted line shows the FWHM of the transmission profile (as in Fig. 18), which by comparison typically encloses approximately $65 \%$ of the overall transmittance.

in Paper I) We also confirm that the magnitude of the ghost profile for a interference filter with $30 \%$ peak transmission, is less than $1.5 \%$ over the full IBIS wavelength range.

Finally, we note that the coating reflectivity remains elevated to even shorter wavelengths than originally specified and the usable range of the instrument may extend down past the nominal operating range to as low as $5500 \AA$.

\section{Effects of instrumental profile on observed spectra}

As stated in the introduction, an accurate knowledge of the instrumental profile is important when applying analysis techniques to acquired data. To quantify the uncertainties that can result if the instrumental profile is not well known, we briefly examine the modifications of some spectral features when two possible instrumental profile are applied. One instrumental profiles 


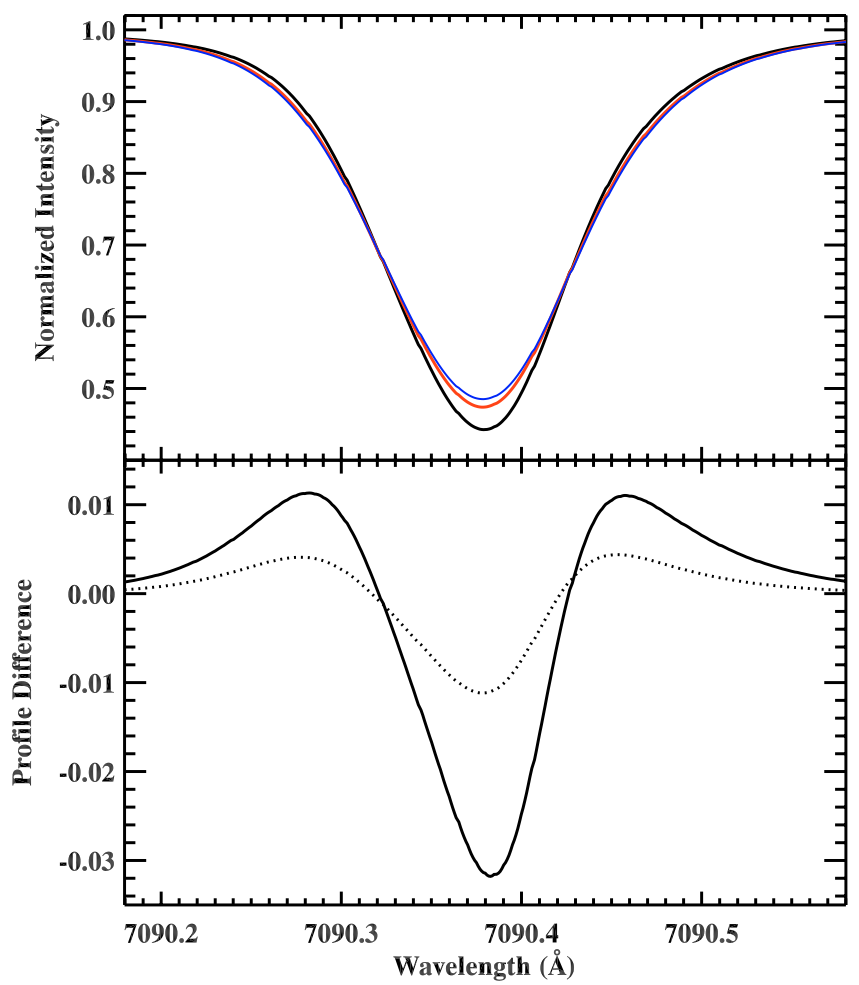

Fig. 21. Top: The effect of the instrumental profile on the synthetic Fe I $7090 \AA$ spectral line profile. The deepest line is the average profile from the simulations. The shallower red and blue lines are the averages after convolution with the instrumental profile for the nominal FPI parameters $(R=0.952$, flatness $=\lambda / 168$ at $7090 \AA)$ and for the full $45 \mathrm{~mm}$ interferometer aperture. All profiles are normalized to the continuum intensity at $7090.1 \AA$. Bottom: The difference between the average synthetic spectral line and the average line after convolution with the nominal profile (solid line) as well as the difference between the profiles resulting from the convolution of the nominal and $45 \mathrm{~mm}$ aperture instrumental profile (dotted line).

is calculated based simply on the nominal characteristics specified by the manufacturer (this profile will be symmetric since there is no assumption about any large-scale spacing defects). At the other extreme, we use the instrumental profile calculated for a $45 \mathrm{~mm}$ aperture using the full distribution of measured errors, resulting in the most asymmetric profile.

For the first test we used synthetic profiles of the Fe I $7090 \AA$ spectral line, obtained from 6 snapshots of a quiet sun hydrodynamic simulation, covering a $6 \mathrm{Mm} \times 6 \mathrm{Mm}$ area in the solar photosphere. To simulate the observations, each synthetic line profile has been convolved with the two different instrumental profiles and then averaged over the full area of the simulations. As is well known, the difference between the synthetic and the "observed" line profiles can be of the order of several percent (Fig. 21). It is notable, however, that the difference between the "observed" profiles, calculated with two plausible instrumental profiles, is reduced only by a factor of 2-3 compared to the differences with the unconvolved synthetic profile. The differences, while small, may be important for some purposes, such as when comparing observed and synthetic profiles at high precision in order to determine abundances.

A second example is shown in Fig. 22 for a similar analysis performed on synthetic Stokes $I$ and $V$ profiles in the Fe I 6301.5 and $6302.5 \AA$ spectral lines calculated for a single position on the Sun. The convolution was performed separately for $I+V$ and $I-V$, and the Stokes $V$ profile was obtained from the

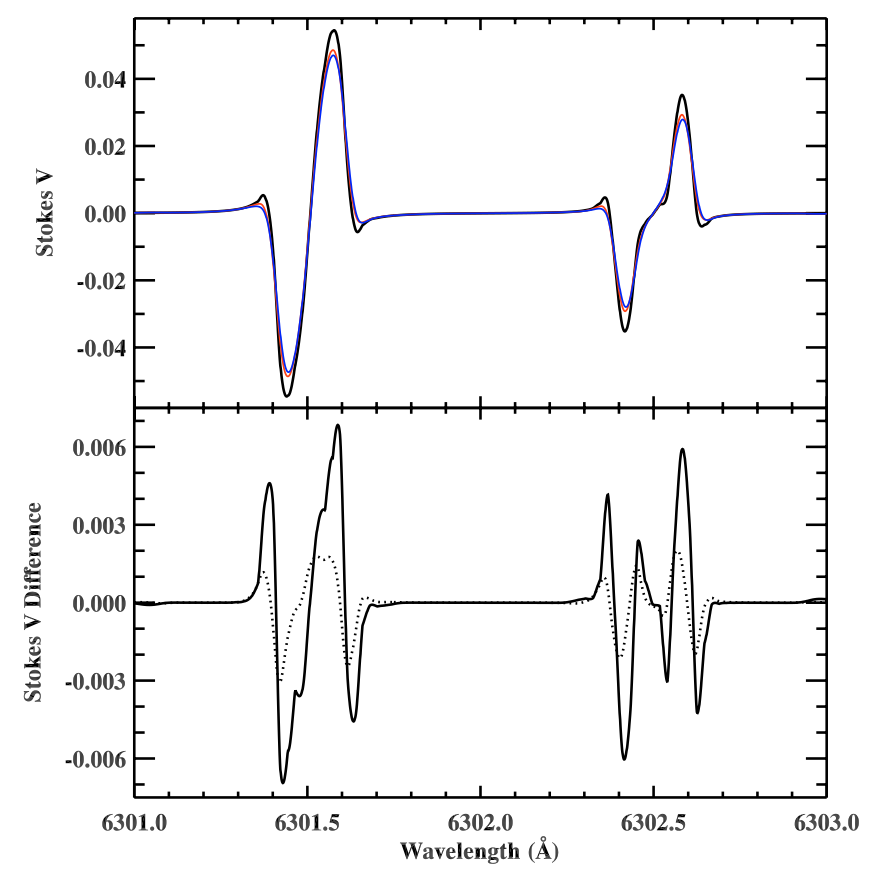

Fig. 22. Similar to Fig. 21, except examining the effect of the instrumental profile on the Stokes $V$ profile. Top: A synthetic Stokes $V$ profile for the $6301.5 \AA$ and $6302.5 \AA$ spectral lines unconvolved by any instrumental profile (solid line) together with the Stokes $V$ profile determined after convolving the measured $I+V$ and $I-V$ spectra with the nominal (red) and $45 \mathrm{~mm}$ aperture (blue) instrumental profiles. Bottom: The difference between the synthetic Stokes $V$ profile and the Stokes $V$ profile calculated after the convolution with the nominal instrumental profile (solid line) and the difference between the Stokes $V$ profiles resulting from the convolution of the nominal and $45 \mathrm{~m}$ aperture instrumental profile (dotted line).

difference of these two signals. The synthetic Stokes $V$ profile has a maximum amplitude of approximately 0.05 (for a continuum intensity of unity), and the convolution with the nominal instrumental profile causes changes at the level of 0.005 , or approximately one-tenth of the original magnitude. The difference between the Stokes $V$ profiles calculated using the two instrumental profiles is again smaller, but still of the order of $2 \times 10^{-3}$, which may be significant for some types of detailed polarimetric analyses. These effects, which are systematic and roughly proportional to the amplitude of the input signal, combined with the uncertainties in the form of the instrumental profile, may in some cases provide the effective lower limit on the overall accuracy of the Stokes profile measurements that can be achieved.

\section{Conclusions}

In this paper we analyzed laboratory measurements made with a frequency-stabilized He-Ne laser in several different instrumental layouts in order to fully characterize the two Fabry-Perot interferometers used in the IBIS instrument. We were able to estimate the effective coating reflectivity of each FPI as well as a reasonably complete description of the cavity errors over all spatial scales. The values we find are different enough from the nominal values provided by the manufacturer to result in significant differences in the instrumental performance characteristics.

The reflectivity of the coating at the laser wavelength is found to be 0.935 and 0.931 for FPI \#1 and FPI \#2 (Sect. 3.2), versus the nominal value of 0.942 . The explanation for this significant difference requires further investigation. We find instead 
that the coating absorption was consistent with the specification of $A \leq 0.002$.

In our analysis, we separated the measured cavity errors into their randomly distributed and large-scale components. The former are presumably due to roughness on the plate surfaces due to polishing or coating defects, and no information was provided by the manufacturer in this regard. We find these defects can be characterized for both FPI by a normal distribution with a standard deviation $\sigma_{t}$ of approximately $9 \AA$.

The large-scale errors could instead be the result of errors in the figuring of the plates, distortions caused by the plate mounting, or due to the stresses in the coating. Typical plate flatness of both FPI due to large-scale errors was estimated by the manufacturer to be $\lambda / 150$ at $6328 \AA$, but we find that this quality is only obtained for the IBIS interferometers over the central $\sim 35 \mathrm{~mm}$ of the aperture. We find in fact that the cavity spacing increases, roughly quadratically, with the radial distance from the center of the interferometer plates, so that the actual magnitude of the cavity errors we measure is $\sim \lambda / 60$ over the full FPI aperture (Sect. 4). Given the similarities between the shapes of the cavity error maps for our two FPI, as well as measurements of other interferometers by the same manufacturer which show a similar distribution (Gullixson 1998; Cao et al. 2004; Denker \& Tritschler 2005), we expect that such a distribution of large-scale cavity errors is a common feature of interferometers manufactured in a similar manner.

We then utilized the fitted maps of large-scale errors and the statistical description of the distribution of roughness errors to compute the instrumental transmission profile for the two FPI in series in a classic mount. We are able calculate this profile for any wavelength and for any illuminated area. We used this calculated profile to determine certain instrumental characteristics of IBIS, such as the spectral resolving power, the parasitic light, and the profile asymmetry. The results we obtained are all well within the design values. We performed tests applying different possible transmission profiles to synthetic solar spectral lines. Comparing the effects of different possible transmission profiles shows that uncertainties in the instrumental profile can produce variations in the simulated profiles that are only two to three times smaller that the errors resulting from not applying any instrumental profile at all.

The measured distribution of the large-scale cavity errors is highly asymmetrical, which, for a FPI used in a classic mounting, will result in an asymmetry of the transmission profile. This will in turn carry over as a distortion in the shape of the observed line profiles that may confuse their interpretation. The large-scale errors will also result in mutual masking between the two FPI and a spatial distribution of the local transmission across the pupil plane producing apodization effects. This masking will occur in a telecentric mount as well, but in this case will result in a variation in transmission across the image plane and in corresponding variations in the shape and wavelength position of the instrumental profile across the field of view (Kentischer et al. 1998).

On a more general note, we stress that the presence of largescale errors whose magnitude is comparable or greater than the magnitude of the roughness errors has important implications for the utilization of the FPI in bidimensional spectrometers. For example the flattening of the plates to remove the effects of non-parallelism will depend on the exact illuminated area of the FPI. Techniques using subapertures of the FPI (Jones \& Bland-Hawthorn 1998; Mickey 2004) may not result in the optimal setup conditions for the interferometer. Similarly, the determination of the tuning parameters for the two or more interferometers in series, by maximizing the overall throughput for example, will depend on the area of the FPI used in the calibration process. Instrumental calibration procedures, often using laser or continuum sources, should be designed to use the same area of the FPI as the observations themselves. We note also that the tuning process could be designed to optimize for parameters other than the overall throughput. For example, it would be possible to perform a tuning that minimizes the mutual masking at the edges of the FPI in order to reduce pupil apodization effects in a classic mount.

Instruments based on Fabry-Perot interferometers are planned for future large-aperture solar telescopes. They will require interferometers with apertures well over $100 \mathrm{~mm}$, and the distribution of surface defects will be an important element in determining whether an instrument meets its operational requirements. Since the cost and technical difficulty rise dramatically with increasing FPI size, it will be necessary for future instruments to use a large percentage of the interferometer aperture, and precise characterization will be necessary to achieve the design goals of future instruments while minimizing costs.

Acknowledgements. We thank C. Denker for making his Zernike fitting code available. We thank M. Asplund for providing the hydrodynamical numerical simulations and A. Asensio-Ramos for performing the radiative transfer calculations. We thank H. Socas-Navarro for providing the synthetic Stokes profiles. Finally, we are grateful to G. Cauzzi for her careful readings of the manuscript and to Tito Grisendi for his priceless assistance in obtaining these data.

IBIS has been designed and constructed by the INAF/Osservatorio Astrofisico di Arcetri with contributions from the Università di Firenze and the Università di Roma "Tor Vergata". IBIS construction and operation has also been supported by the Italian Ministero dell'Università e della Ricerca (MIUR) as well as the Italian Ministry of Foreign Affairs (MAE).

We thank the National Solar Observatory staff for all their assistance in the operation of IBIS. National Solar Observatory is operated by the Association of Universities for Research in Astronomy, Inc. (AURA), under cooperative agreement with the National Science Foundation.

\section{References}

Anderson, D. J. 1995, Appl. Opt., 34, 8062

Beckers, J. M. 1998, A\&AS, 129, 191

Bendlin, C., \& Volkmer, R. 1995, A\&AS, 112, 371

Burgh, E. B., Nordsieck, K. H., Kobulnicky, H. A., et al. 2003, in Instrument Design and Performance for Optical/Infrared Ground-based Telescopes, ed. M. Iye, \& A. F. M. Moorwood, SPIE Conf. Proc., 4841, 1463

Cao, W., Denker, C. J., Wang, H., et al. 2004, in Telescopes and Instrumentation for Solar Astrophysics, ed. S. Fineschi, \& M. A. Gummin, Proc. SPIE, 5171, 307

Cauzzi, G., Asensio Ramos, A., Reardon, K., \& Janssen, K. 2006, in Solar MHD Theory and Observations: A High Spatial Resolution Perspective, ed. J. Leibacher, R. F. Stein, \& H. Uitenbroek, ASP Conf. Ser., 354, 26

Cavallini, F. 1998, A\&AS, 128, 589

Cavallini, F. 2006, Sol. Phys., 236, 415

Cepa, J., Aguiar-Gonzalez, M., Bland-Hawthorn, J., et al. 2003, in Instrument Design and Performance for Optical/Infrared Ground-based Telescopes, ed. M. Iye, \& A. F. M. Moorwood, SPIE Conf. Proc., 4841, 1739

Denker, C., \& Tritschler, A. 2005, PASP, 117, 1435

Denker, C. J., Ma, J., Wang, J., et al. 2003, in Innovative Telescopes and Instrumentation for Solar Astrophysics, ed. S. L. Keil \& S. V. Avakyan, SPIE Conf. Proc., 4853, 223

Elson, J. M., Rahn, J. P., \& Bennett, J. M. 1980, Appl. Opt., 19, 669

Gullixson, C. 1998, Characteristics of Available Fabry-Perot Filters, Tech. Rep., National Solar Observatory, Sunspot, NM

Ishigaki, T., Hayashi, T., Ohtani, H., et al. 2004, PASJ, 56, 723

Jones, D. H., \& Bland-Hawthorn, J. 1998, PASP, 110, 1059

Kentischer, T. J., Schmidt, W., Sigwarth, M., \& Uexkuell, M. V. 1998, A\&A, 340,569 
Mack, J. E., McNutt, D. P., Roester, F. L., \& Chabbal, R. 1963, Appl. Opt., 2, 873

Mahapatra, D. P., \& Mattoo, S. K. 1986, Appl. Opt., 25, 1646

Martinez-Herrero, R., Mejias, P. M., \& Bernabeu, E. 1985, Appl. Opt., 24, 315

Mickey, D. L. 2004, Sol. Phys., 220, 21

Netterfield, R. P., \& Ramsay, J. V. 1974, Appl. Opt., 13, 2685

Puschmann, K. G., Kneer, F., Seelemann, T., \& Wittmann, A. D. 2006, A\&A, 451, 1151

Ramsay, J. V. 1969, Appl. Opt., 8, 569

Ramsay, J. V., Kobler, H., \& Mugridge, E. G. V. 1970, Sol. Phys., 12, 492

Scharmer, G. B. 2006, A\&A, 447, 1111
Skumanich, A., \& Lites, B. W. 1987, ApJ, 322, 473

Socas-Navarro, H. 2001, in Advanced Solar Polarimetry - Theory, Observation, and Instrumentation, ed. M. Sigwarth, ASP Conf. Ser., 236, 487

Socas-Navarro, H. 2003, Neural Networks, 16, 355

Vaughan, J. M. 1989, The Fabry-Perot interferometer. History, theory, practice and applications (The Adam Hilger Series on Optics and Optoelectronics, Bristol: Hilger)

von der Lühe, O., \& Kentischer, T. J. 2000, A\&AS, 146, 499

Wilksch, P. A. 1985, Appl. Opt., 24, 1502

Yoshikawa, I., Kameda, S., Matsuura, K., et al. 2007, Planet. Space Sci., 55, 1622 